Microseismic Data Acquisition, Processing, and Event Characterization at the Illinois Basin - Decatur Project

Robert Will $^{\text {a }}$, George El-Kaseeh ${ }^{\mathrm{b}}$, Paul Jaques ${ }^{\mathrm{c}}$, Michael Carney ${ }^{\mathrm{b}}$, Sallie Greenberg ${ }^{\mathrm{d}}$, and Robert Finley ${ }^{\mathrm{d}}$

${ }^{a}$ Schlumberger Carbon Services, 1875 Lawrence St. Suite 810, Denver, CO 80202

${ }^{\mathrm{b}}$ Schlumberger Carbon Services, 14090 Southwest Freeway Suite 240, Sugar Land, TX 77478

${ }^{\mathrm{c}}$ Schlumberger Madingley Road, Cambridge, CB3 0EL, UK

${ }^{\mathrm{d}}$ Illinois State Geological Survey, University of Illinois at Urbana-Champaign, 615 East Peabody Drive, Champaign, IL 61821

${ }^{\dagger}$ Corresponding author:

Robert Will

Schlumberger Carbon Services

1875 Lawrence St. Suite 810

Denver, CO USA

80202

Tel.: +1-303-244-8240

E-mail address: RWill@ slb.com 


\title{
Microseismic Data Acquisition, Processing, and Event Characterization at the Illinois Basin - Decatur Project
}

Robert Will $^{\text {a }}$, George El-Kaseeh ${ }^{\mathrm{b}}$, Paul Jaques ${ }^{\mathrm{c}}$, Michael Carney ${ }^{\mathrm{b}}$, Sallie Greenberg ${ }^{\mathrm{d}}$, and Robert Finley ${ }^{\mathrm{d}}$

\begin{abstract}
Microseismic monitoring at the Illinois Basin - Decatur Project (IBDP) is accomplished using a combination of commercially available components that are integrated to provide real-time analysis and remote processing capabilities by means of a purpose-built data management system and secure web portal. The processing workflow consists of real-time, remote access, and inhouse processing components, which provides a seamless path from in-field quality control to final locations with short turn-around times. Event location is performed using an adaptation of the Geiger method, which is designed to be robust for the sparse but localized observation sets typically encountered with injection monitoring. Observed microseismicity displayed distinct linear clustering and increased in distance from the injection well over time, presenting challenges for location accuracy of more distal events. The velocity model, a key component in event location and characterization, evolved through early stages of the project as new wells were drilled providing the opportunity for improved observation geometry and acquisition of additional controlled energy source points for model calibration. The experience gained at IBDP highlights the importance of field systems and processing flows that allow adaptation to evolving operational conditions and microseismic event activity.
\end{abstract}

\section{Keywords}

Microseismic, acquisition, processing, Geiger, trigger, mechanism

\section{Introduction}

The Illinois Basin - Decatur Project (IBDP) is a United States Department of Energy - National Energy Technology Laboratory-funded carbon capture and storage (CCS) project that, in November 2014, successfully completed its goal of safely injecting one million tonnes of carbon dioxide deep into the Mt. Simon Sandstone. This paper will describe the microseismic acquisition systems, the processing flow, and the results achieved.

Passive microseismic monitoring networks, such as the one deployed at the IBDP site, continuously record vast amounts of passive seismic data containing information about ground motion. Data streams include both noise and coherent signals emitted by discrete subsurface displacement events.

Coherent seismic signals may be used to estimate the time, location, and other physical characteristics of the displacement mechanisms responsible for the energy emission. These "focal mechanisms" are parameterized using rock mechanics theory in which emitted energy characteristics are functions of in-situ stress magnitude and orientation, rock properties, 
displacement distance and direction, stress drop, and other properties of the displacement surface. Under favorable conditions event location and some focal mechanism characteristics may be estimated from analysis of the coherent energy emissions.

Accurate location of microseismic source events and estimation of focal mechanism characteristics from passive seismic data are achieved through a series of data manipulations (processing flow) that combines seismic signal processing, seismology, and fault mechanics theory. The processing flow may be broken into the main steps of data preparation, event detection/location, and focal mechanism characterization. In addition, location and characterization of microseismic events requires a velocity model that serves as a framework for many data processing computations and the components of which are calibrated using controlled or known source events.

While the final velocity model uses Vertical Transverse Isotropic (VTI) anisotropy structure with Thomsen parameter formulation, multiple velocity models have been used throughout the life of the project owing to the addition of new array components and calibration data. This has provided valuable insight into the sensitivity of processing results with respect to both the velocity model and the observation network geometry.

The data processing flow was followed by statistical and graphical trend analysis of the characterized microseismic events. It will be shown that simple graphical and statistical trend analyses reveals coherent features that are consistent with the geologic and geomechanical site characterization. These observed trends formed the rationale for implementation of microseismic results in subsequent coupled hydro-mechanical modeling work.

\section{Acquisition Systems}

2.1. Overview

The IBDP microseismic monitoring network is comprised of multiple subsurface arrays deployed in three separate wells with data streams integrated through a combination of their associated Data Acquisition Units (DAU's) and a central integration computer. All borehole arrays are geophone based. The Sercel Slimwave is a digital multi-level array. Field filtering, digitization, and recording are performed within the respective DAU's.

The subsurface data acquisition network is made up of the following (Figure 1):

- Injection Well (CCS1)

○ A 2-level array of a 4-component WellWatcher PS3* passive seismic sensing system.

- Geophysical Monitoring Well (GM1)

- A 31-level array of 3-component high temperature geophones.

- The Illinois Industrial Carbon Capture and Storage Project's Verification Well (VW2)

- A 5-level array of a 3-component multi-level downhole seismic array had been temporarily deployed in VW2. 
Data recording, system integration, and real-time data acquisition processes are performed through the following surface systems (Figure 2) located at the field site;

- $\quad$ CCS1 and GM1

- Data from both CCS1 and GM1 subsurface arrays are recorded using a 96 channel DAU located at the field office near CCS1.

- VW2

- Data from the VW2 subsurface array is recorded using a 24 channel DAU located at the wellhead.

- Data Integration System

- Data from all subsurface arrays are integrated and real-time data acquisition functions are performed using a microseismic integrated data acquisition system located at the field office.

In addition to the above integrated borehole monitoring configuration, five surface seismometer stations have been installed by the Illinois State Geological Survey.

As part of the control and monitoring of injection operations, a real-time acquisition and control software was used to coordinate the collection and access to the extensive non-seismic data (pressure, temperature, rate, etc.) from the injection well (CCS1), monitoring well (VW1) and the surface facilities.

\subsection{System Description}

It is tempting to view the current IBDP microseismic monitoring network as a static configuration designed to achieve a specific, pre-determined set of technical objectives. In reality the system configuration has evolved throughout the life of the project and, at any given time, reflects the best possible solution to multiple and evolving technical challenges under an ever changing set of operational and commercial conditions and constraints. The following sections describe how each system component has been designed and/or adapted to meet these challenges. 


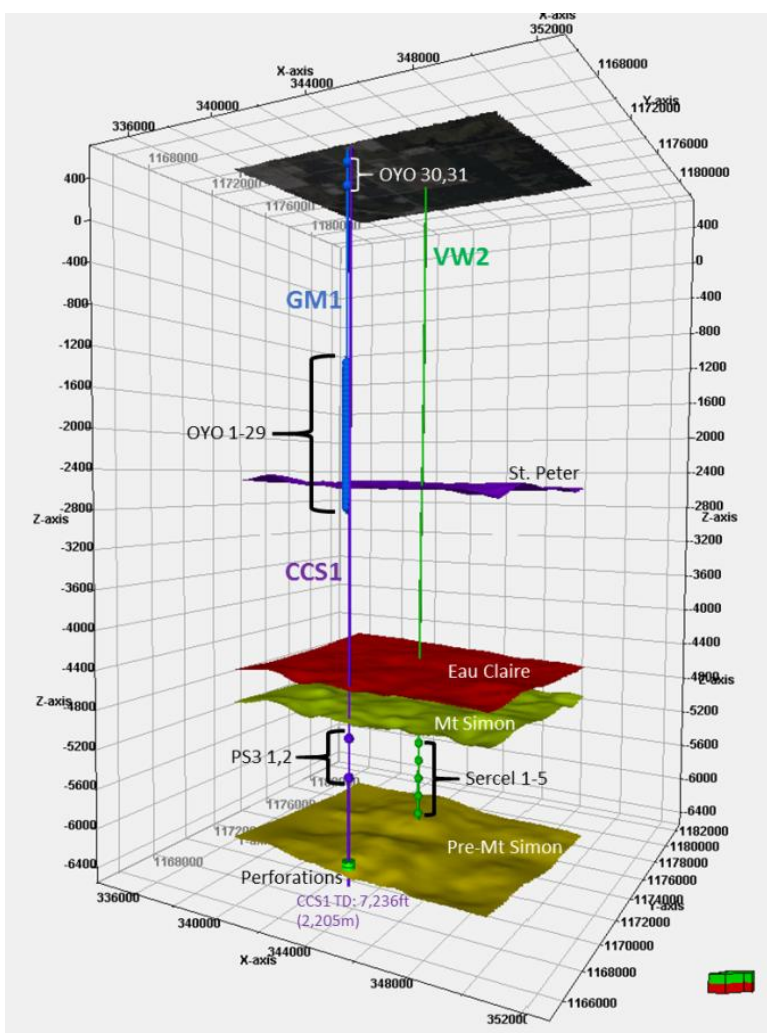

Figure 1 - Subsurface array configuration. Distance units are feet, $\mathrm{Z}$ axis is referenced to mean sea level.

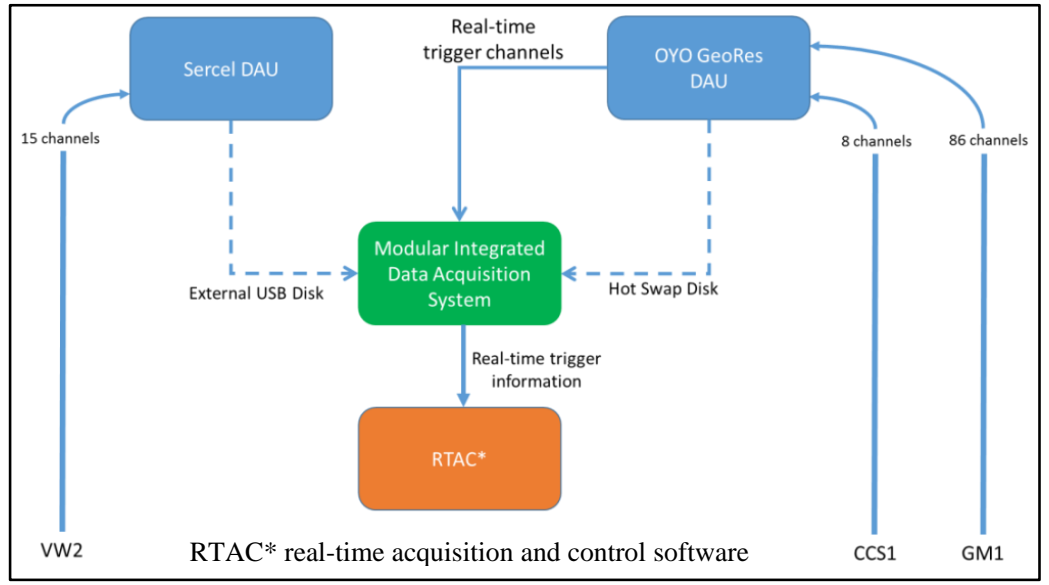

Figure 2 - Data acquisition and integration system configuration.

\section{The Injection Well - CCS1}

The 4-level WellWatcher PS3 array in CCS1 was deployed in 2009 during completion of the well. Pre-injection data acquisition began in May 2010. In the original monitoring plan these tools were anticipated to provide the majority of the observations for microseismic location and characterization. The decision to deploy this technology was driven by two key technical factors: 
- The availability of a specialized deployment mechanism (Figure 3) which effectively decouples sensors from the injection tubing string thereby reducing flow induced noise coupling to the sensors.

- The use of 4 tetrahedral oriented sensors in each tool allowing reconstruction of the 3-component wavefield, should any single sensor fail.

- The tetrahedral configuration is the optimal four-sensor configuration.

- Single sensor malfunction still allows the full three-dimensional (3D) wavefield to be recorded.

- The tetrahedral sensor configuration allows for sample-by-sample data QC for efficient system performance monitoring.

- Frequency ranges where vector fidelity is poor are easily identified and so can be excluded from data analysis.

- Long-term sensitivity drift or performance change is easily diagnosed and can be corrected for in situ.

- The coherency function can be used as an aid to phase identification.

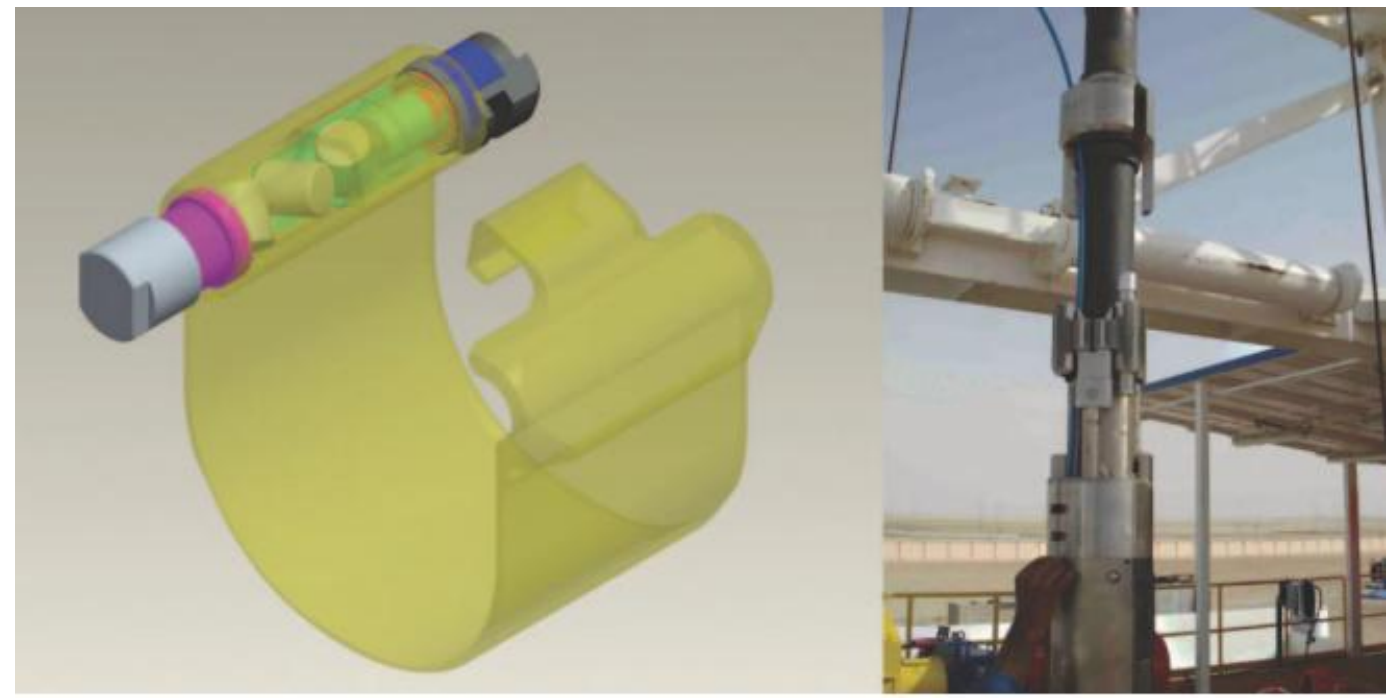

Figure 3 - Tetrahedrally arranged sensors used for micro-seismic data acquisition on left. The clamp before it is deployed on tubing with the geophone on the right.

The four sensor levels in CCS1 were set at subsurface depths spanning the interval from the Eau Claire Shale to the Mt. Simon A Formation, intended to provide reasonable signal fidelity and location accuracy throughout the primary seal and injection intervals. Damage during deployment and poor coupling compromising data from the shallowest two sensors resulted in reduction of the CCS1 array to the two sensors located nearest to the injection zone (Table 1). The eight channels of data from these two sensors are recorded via the 96 DAU located at the nearby field office. 


\section{The Geophysical Monitoring Well - GM1}

A 31-level array of 3-component tools was permanently cemented into place (outside of casing) in GM1 during well construction in 2009. Recording commenced in May 2010. The primary objective of the GM1 array was for use in the planned high repeatability time-lapse 3D vertical seismic profile (VSP) surveys for plume monitoring. As such, the primary well construction and array design (Table 2) considerations were linked to VSP imaging objectives, with support of the microseismic monitoring array in CCS1 as a secondary objective. However, given the reduction of observations in CCS1 discussed above, the significance of observations from the GM1 array for microseismic characterization greatly increased. Data from a selected 28-level (86-channel) subset of the 31-level array are recorded to the 96 DAU along with data from CCS1. The data was subsampled from the full 31-level array due to loss of fidelity in several of the geophone levels.

\section{The Verification Well - VW2}

The VW2 array was initially deployed in September 2013 and was added to the network in response to the widening footprint of microseismic activity presenting need to supplement the observation geometry and removed in February of 2015 in preparation for later deployment in a new location. The VW2 array is a removable deployment with the five 3-component tools located between 6,150 feet (1874.52 meters) and 6,950 feet (2118.36 meters), at 200 foot (60.96 meters) intervals and coupled to the casing with a mechanical stand-off arm mechanism. Data from the VW2 array are recorded at the wellhead using the dedicated DAU.

\section{Data Acquisition Units}

\section{$\underline{\text { CCS1 and GM1 }}$}

A 96 channel DAU is used to collect and record data from receiver arrays in CCS1 and GM1 into a single SEG-2 file. This merging of datasets was operationally expedient due to the proximity of these two wells. Eight channels from the two active CCS1 arrays (each 4-component) are combined with 86 selected channels from the GM1 array for a total of 94 input channels. Data from both systems are filtered and sampled using the same system parameters (Table 1).

\begin{tabular}{|l|l|}
\hline Total number of channels & 96 \\
\hline Record length & 10 seconds continuous recording \\
\hline Sample rate & 0.5 Millisecond \\
\hline Filter & $\begin{array}{l}\text { Low cut filters: Digital 3Hz } \\
12 \mathrm{~dB} / \text { Oct / Analog 8Hz }\end{array}$ \\
\hline Data format & SEG-2 \\
\hline Time stamp & $\begin{array}{l}\text { Global Positioning System (GPS) } \\
\text { time synchronization }\end{array}$ \\
\hline Number of files & 8640 files per day \\
\hline
\end{tabular}

Table 1 - CCS1 and GM1 array data recording parameters. 
VW2

Data from the retrievable array in VW2 are recorded using a stand-alone DAU located in an enclosure near the wellhead and configured as listed in Table 2.

\begin{tabular}{|l|l|}
\hline Total number of channels & 15 \\
\hline Record length & 30 seconds continuous recording \\
\hline Sample rate & 0.5 Millisecond \\
\hline Filter & N/A \\
\hline Data format & SEG-Y \\
\hline Time stamp & GPS time synchronization \\
\hline Number of files & 2880 or 2879 files per day \\
\hline
\end{tabular}

Table 2 - VW2 array recording parameters.

\section{Field System Integration}

As seen in the above descriptions, the IBDP observation network is made up of distributed arrays and DAU's with varying specifications. Integration of these components is achieved through a microseismic integrated data acquisition system. This system is purpose-built and specifically designed for the original monitoring network, but with sufficient capacity and flexibility to be adapted to accommodate network expansion such as the addition of the temporary VW2 array.

In addition to managing the real-time recording of data from CCS1 and GM1 arrays, the integration system performs various real-time functions such as event triggering, real-time preliminary event location and magnitude estimates, and automated notifications via email. Data from the remote VW2 array are transferred to the microseismic integrated data acquisition system periodically in coordination with the data processing schedule via USB disk. Precise time registration across all systems is maintained through their respective synchronized Global Positioning System (GPS) clocks. The details of the data management and preliminary processing functions performed within this data system are described in the later discussion of data processing.

Key microseismic data attributes such as triggering information and event detection rates are communicated to the acquisition and control software via the microseismic integrated data acquisition system. The acquisition and control software utilized enables real-time access for both onsite and remote users to time-sensitive data. Built-in graphical analyses and download functions expedite informed operational decisions.

\section{Surface Stations}

In 2013 a network of five 3-component seismometers were installed in shallow, near-surface vaults (locations shown in Figure 4). This network was intended to be a back-up system for the downhole microseismic systems for large magnitude events. The instruments in the near-surface system will continue to record on-scale if a seismic event, large enough to saturate the downhole microseismic systems, were to occur in the vicinity of the IBDP study area.

If a large event were to occur, the sensors in the downhole systems would be able to detect the event and determine its $3 \mathrm{D}$ location, but might not be able to accurately measure the magnitude 
of the event. Less sensitive surface-deployed seismometers were chosen to provide coverage in this range of larger seismic amplitude. Surface seismic networks are a common and inexpensive means of deploying micro-seismic monitoring systems. The surface microseismic stations network also provides a means for verifying the feasibility of surface seismometers and comparing their effectiveness relative to downhole microseismic systems. While these data are not integrated with downhole data in event processing, the data have been used for independent verification of larger events.

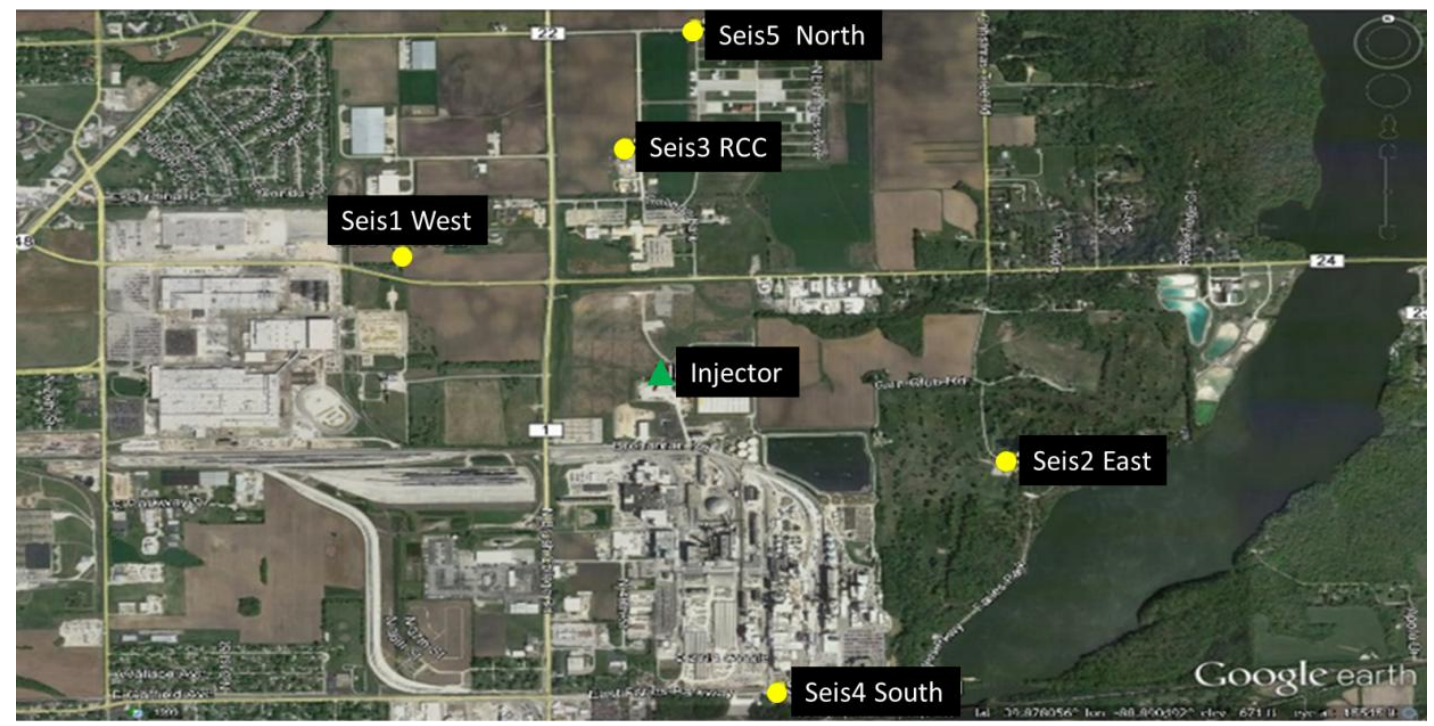

Figure 4 - Location of the five surface seismometers (yellow) and the injector well (green) in Decatur, Illinois.

In mid-2013, four surface seismic stations were deployed in approximately the four cardinal directions from the IBDP CCS1 well at radial distances of 3,500 feet (1066.8 meters) to 6,000 feet (1828.8 meters). Sites were chosen to be on Archer Daniels Midland Company property and away from buildings. The fifth station is located outside the National Sequestration Education Center building at Richland Community College. Real-time data monitoring is performed in a nearby campus facility.

\section{Acquisition Systems}

3.1. Workflow Overview

Figure 5 shows a generalized workflow diagram for microseismic data processing and event characterization. This workflow includes tasks for calibration of the velocity model and determination of in-ground receiver orientations (left) which are performed during project initialization and periodically updated. Routine data processing and event characterization tasks (right-side of figure) will be described in this section. Velocity model calibration and tool orientation will be discussed later in the paper. 


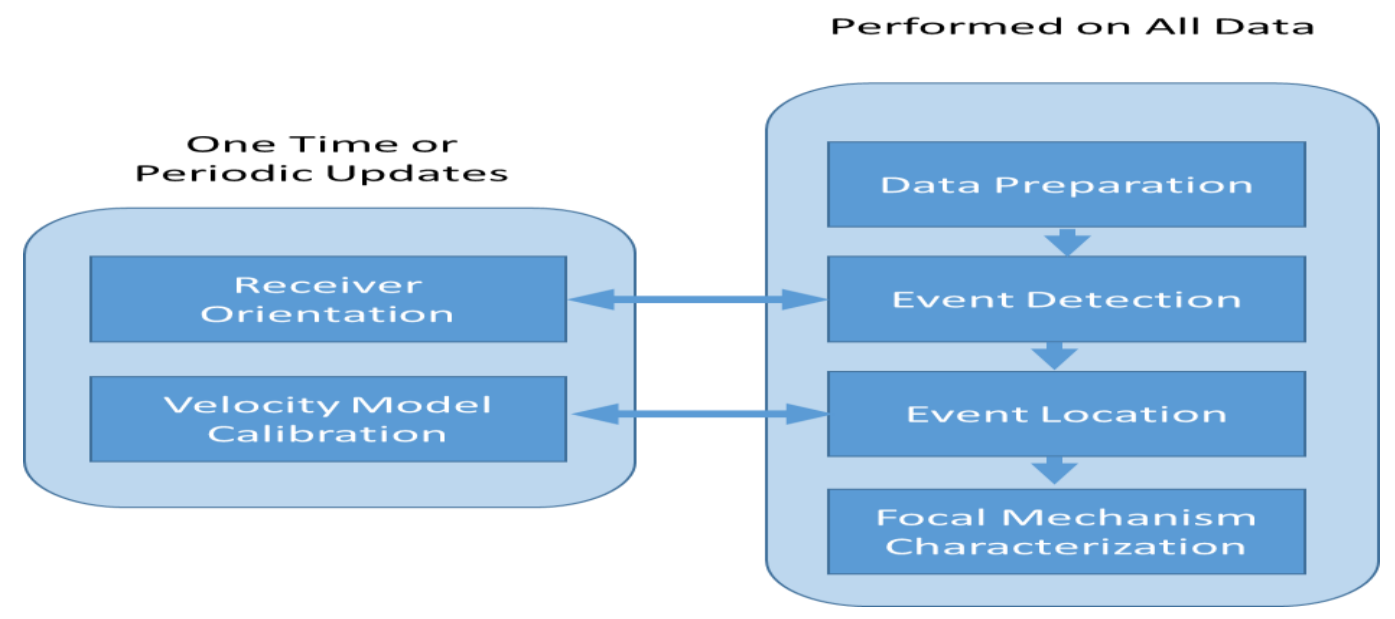

Figure 5 - Processing and characterization workflow.

\subsection{Workflow Overview}

The workflow illustrated above has been implemented at IBDP in a manner that is tailored to the specific acquisition configuration, which meets project objectives with respect to resource allocation and turn-around time for final products. Since the analyst performing event location and characterization is not located at the field site, a combined in-field/in-house workflow (Figures 6 and 7) has been developed whereby the amount of raw data to be transferred for processing is significantly reduced through preliminary infield processing (triggering). Once received at the data center, the raw data for all triggered events are reevaluated for improved location and characterization.

\section{On-Site/In-Field Data Preparation (Figure 6)}

- Transfer monthly 15 channel SEG-Y data from the VW2 DAU to the Microseismic Integrated Data Acquisition System.

- $\quad$ Merge VW2 data with the 94 channel GM1/CCS1 data into a single 109 channel SEG-2 dataset using GPS time (UTC) synchronization.

- Run triggering algorithm on merged SEG-2 dataset to create subset of triggered data in intermediate processing format. Triggered dataset is as small as $2 \%$ of the original dataset depending on activity.

- Transfer raw data subset for triggered events to the data analyst via remote access.

\section{In-House Event Detection and Location (Figure 7)}

- Manually check raw data for all triggered events and remove all false triggers. Manual inspection typically yields approximately $15 \%$ of the triggered dataset as real events.

- Manually inspect each event, picking P, S, polarity and hodograms. Assess whether or not event is located with reasonably low uncertainty. 
- Locate the event using a single fixed start position for Geiger location, check that event locates correctly, i.e. located event has correct azimuth.

- If location is not satisfactory, re-locate the event using a list of predefined starting positions.

\section{Source Characterization}

- Calculate magnitudes using displacement spectral analysis.

- $\quad$ Calculate $\mathrm{P}$ to $\mathrm{S}_{\mathrm{H}}$ wave amplitude ratios for Fault Plane Solution (FPS) analysis.

\section{Quality Control and Final Output}

- $\quad$ Map and quality control for outliers in located position, magnitude, and uncertainty.

- $\quad$ Produce spreadsheet file containing event solution parameters as well as updated cumulative statistical and graphical event analyses.

Event detection and location are largely manual processes and are performed on a monthly basis after tool orientation, velocity model calibration, and filter parameter optimization have been completed or updated. However, real-time functionality has been implemented in the Microseismic Integrated Data Integration System to automatically flag significant events in realtime with a magnitude greater than 0 , triggering an email which signals the need for review.
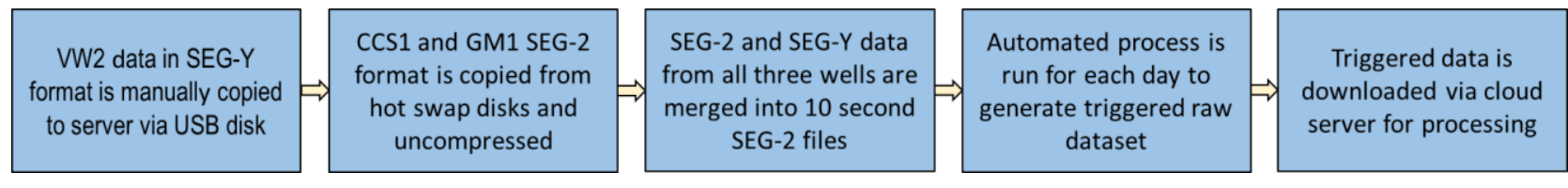

Figure 6 - In-field data preparation process.

Each of these main tasks will be discussed later in the paper with examples from the IBDP dataset.

\section{In-field Data Preparation}

The following process (illustrated in Figure 7) is applied on a periodic basis. Where possible, the process is automated. However, some manual intervention is required.

- At the end of each (UTC) day, the 8640 ten second files recorded at the VW2 DAU are compressed at the DAU.

- $\quad$ SEG-2 data from CCS1 and GM1 is stored in one folder for each day.

- For each of the 8640 SEG-2 files containing 94 channels, the corresponding 15 channels from the SEG-Y files are merged in order to create a new folder containing 8640 SEG-2 files with 109 channels.

- The merged 109 channel SEG-2 files are input to the triggering process. 
- A subset of raw data is created for the raw data falling within triggered windows and output in internal processing system format. The final triggered raw dataset is downloaded for processing.

- $\quad$ CSV files containing root mean square (RMS) values are generated for loading into Excel to create monthly plots of the tool performance.

- All intermediate process datasets are deleted.

At the end of each month, the entire data set is transmitted to the data center for final event detection location and characterization.

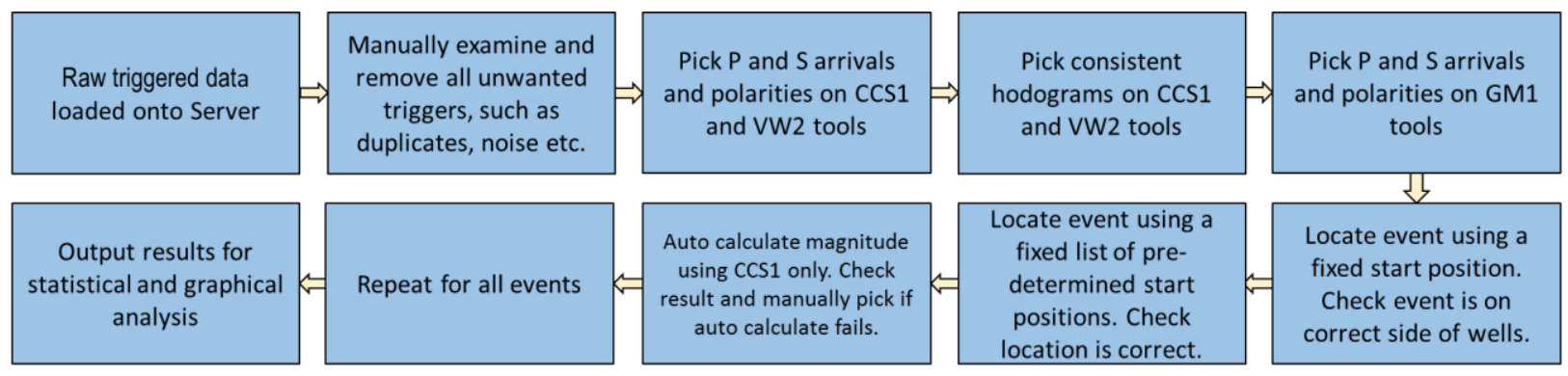

Figure 7 - In-house portion of the microseismic processing and characterization workflow as implemented at IBDP.

\section{Triggering/Event Detection}

\subsection{Triggering Parameters}

The automated triggering process is applied as follows;

a) Create a raw data subset containing only designated trigger channels (eight CCS1 channels plus six channels from the top and bottom tools in VW2).

b) Apply an 80 coefficient error prediction filter to eliminate any repetitive signals such as $60 \mathrm{~Hz}$ (and harmonics) from electrical systems and injection related (tubing coupled) noise.

c) Apply a bandpass filter.

d) Calculate a Short Term Average/Long Term Average (STA/LTA) function (referred to as Energy Ratio method).

The STA/LTA data are subjected to the trigger algorithm wherein a minimum of four of the fourteen trigger input channels must exceed a pre-defined STA/LTA function threshold. Once a trigger occurs, a $250 \mathrm{~ms}$ wait time is necessary before another trigger can occur in order to avoid retriggering on the same event. 


\subsection{Triggering Example}

The trigger data preparation process described in steps a) to d) above is illustrated in Figures 8 to 11. Figure 9 shows an example of a typical small event before any filtering has been performed. The top two seismograms in these image sets are from the tools in CCS1. The bottom five seismograms are from the tools in VW2.

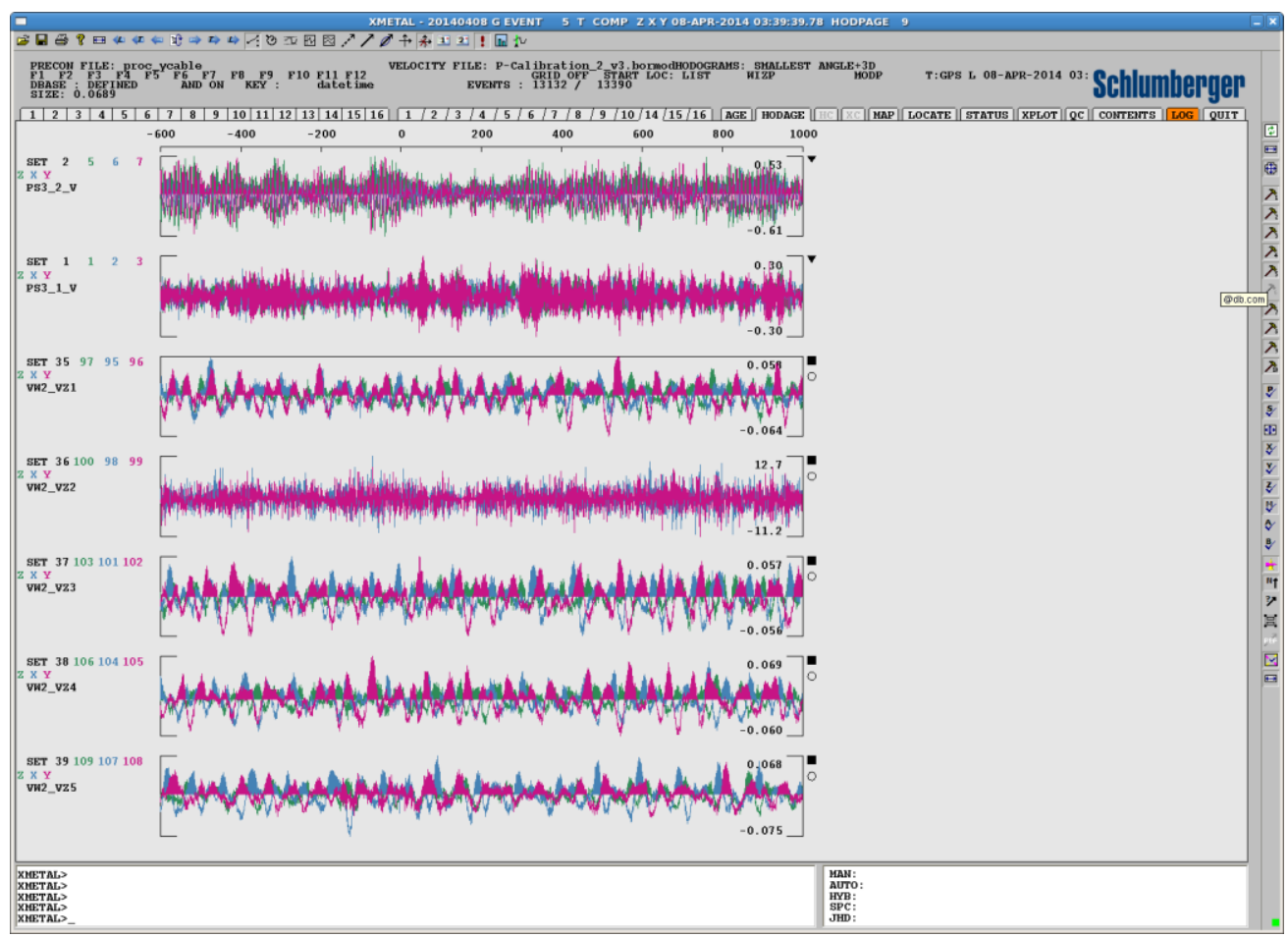

Figure 8 - Typical small event before filtering

Beginning with a dataset shown in Figure 8, Figures 9, and 10 illustrate that events become discernible to the eye on some channels after application of predictive and bandpass filters. Figure 11 shows the result of the STA/LTA calculation which is input to the trigger algorithm to determine if an event has occurred. 


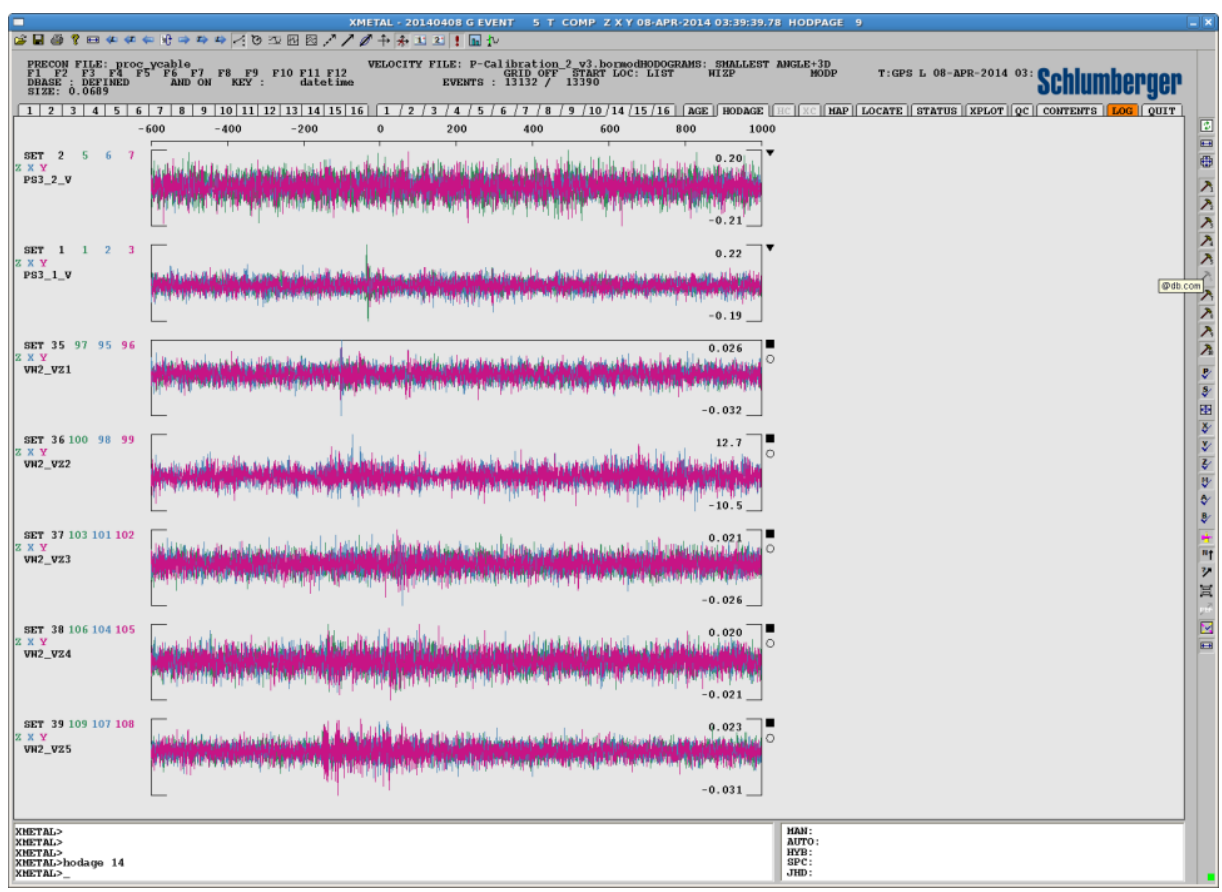

Figure 9 - Data from Figure 8 after application of predictive filtering.

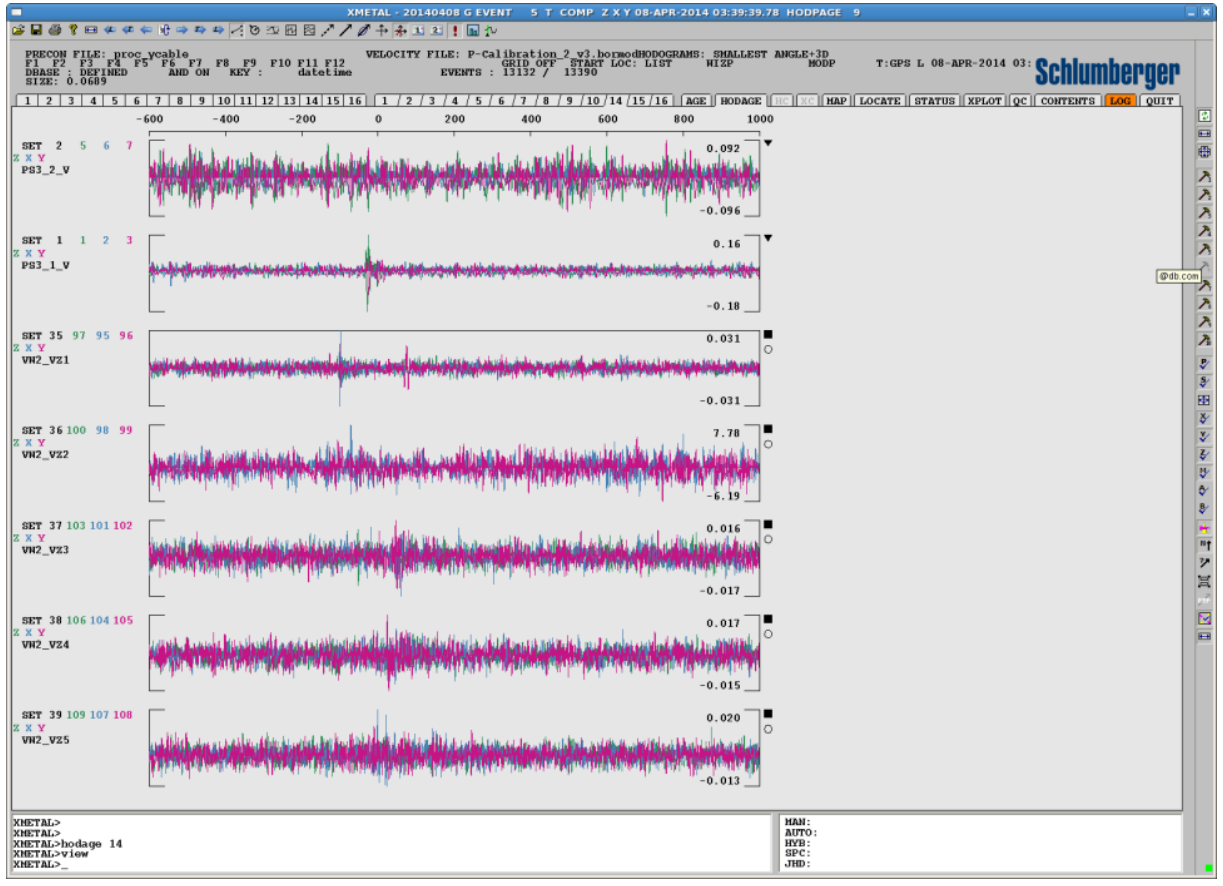

Figure 10 - Data from Figure 9 after application of bandpass filter. 


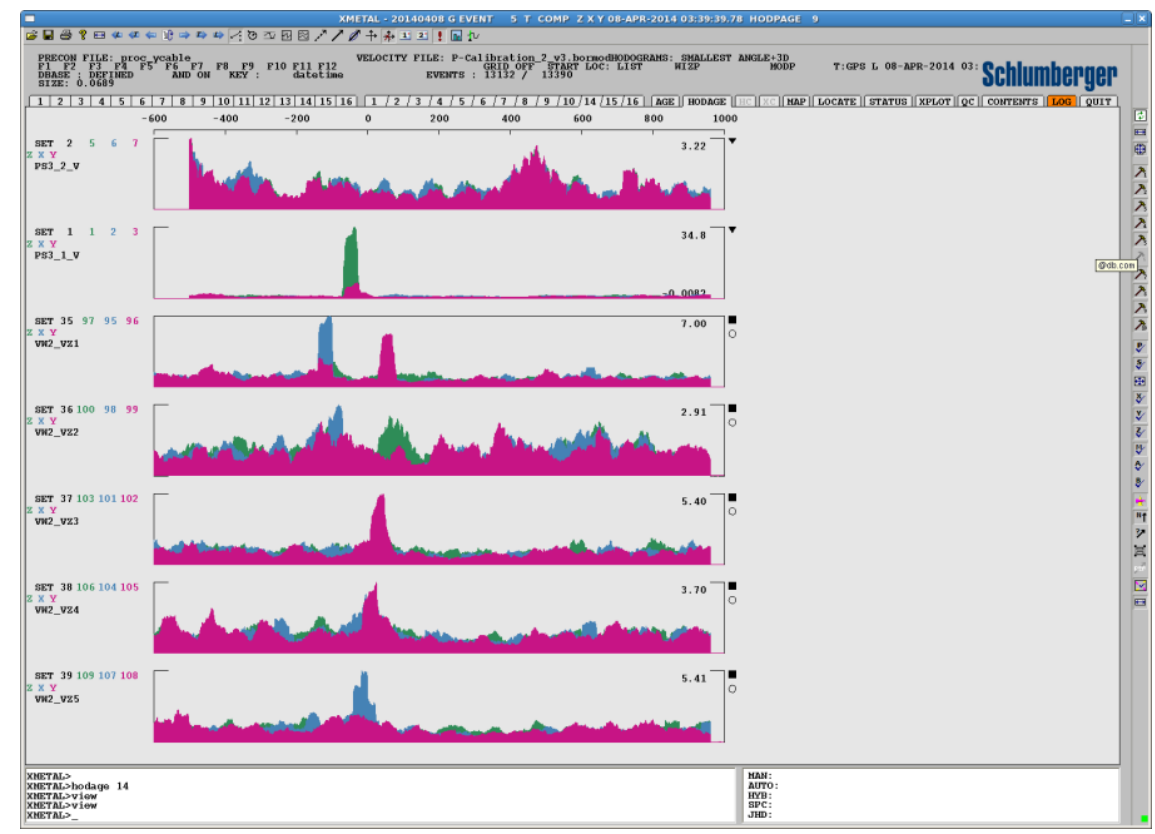

Figure 11 - STA/LTA function used for event trigger computation.

The application of triggering remotely on the data acquisition system results in an approximately 98\% reduction in the amount of raw data that are transmitted to the data center for analysis. After the triggered dataset is transferred to the data center, all triggered event records are reprocessed from raw data using a different filtering scheme and visually inspected. The data are not retriggered. False triggers are eliminated on the basis of raw $\mathrm{P}$ - and S-wave arrival time consistency and signal quality. Of the approximately 128,000 triggered events since the beginning of data acquisition, roughly $15 \%$ have been assessed as real events.

Detecting large events is easy but smaller events are more difficult, particularly when the amplitude of the events is below the level of background noise. The major long term contribution to background noise at this location comes from the constant injection, although road traffic and railways provide an increase in noise levels at certain times of the day (Figure 12).

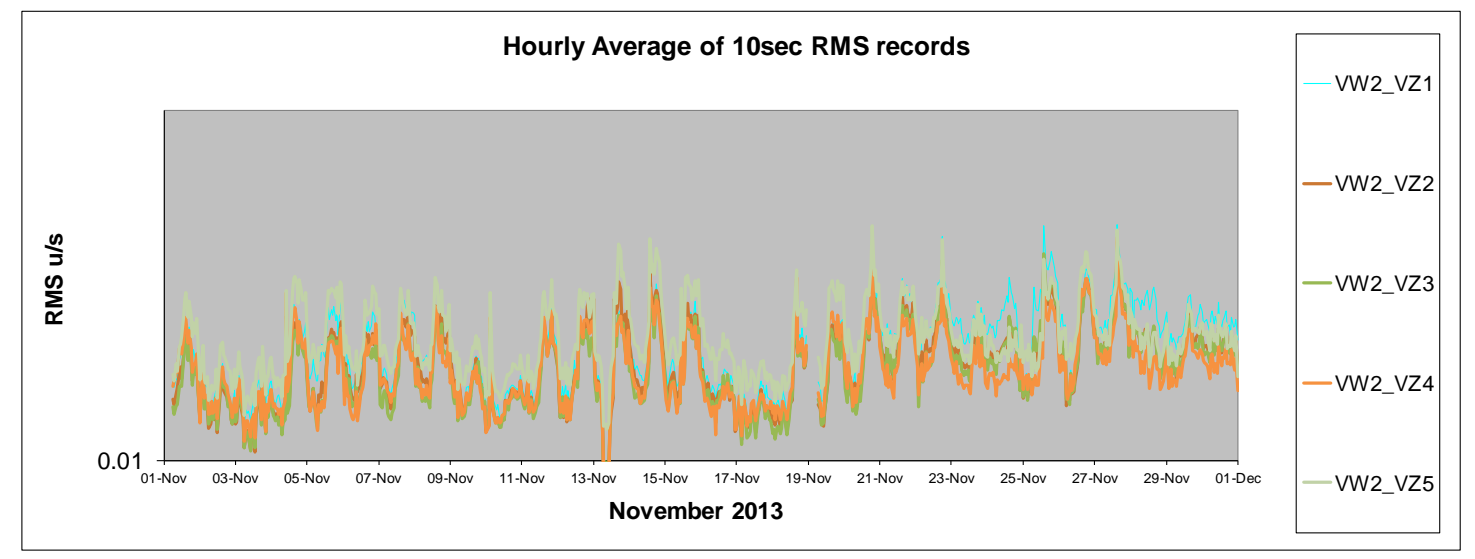

Figure 12 - RMS noise showing daily peaks due to road traffic. 
Figure 13 shows waveforms and hodograms of a real event on the VW2 array selected from the raw triggered event dataset and after pre-filtering. P- and S-wave events are clearly visible on waveforms and the particle motion hodograms on the right side of the figure show excellent linear character.

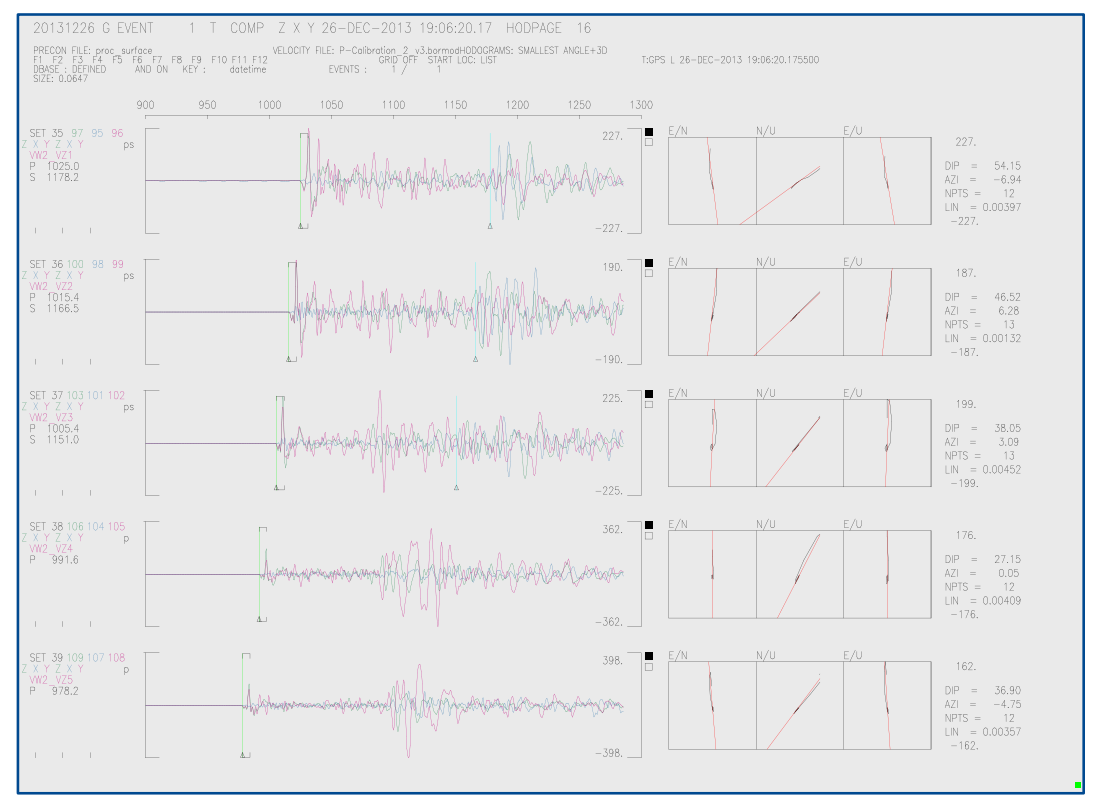

Figure 13 - Example real event waveforms and hodograms after detection and filtering.

\section{Event Location}

Events selected as real events from the triggered dataset are located using a modified form of the Geiger method (Geiger, 1912) in which the wave propagation direction derived from hodograms is used as an additional constraint in the solution.

One of the key challenges for any typical microseismic locating effort is the non-uniqueness of the location solution resulting from sparse observation networks. Although the modification to the Geiger method used at IBDP takes advantage of additional information from the hodogram to constrain the location, non-uniqueness still exists. Such non-uniqueness has been treated through inspection of the solution RMS residual space and careful selection of starting points for the Geiger solution.

Examples of location solution non-uniqueness are illustrated in Figures 14 and 15. In Figure 14 a slice through the solution RMS residual space for a selected event near well VW2 shows two significant minima (deep blue). Without a carefully selected starting point for the Geiger solution there is a high probability of converging on a false minima yielding an incorrect location. Figure 16 illustrates the impact of observation geometry on solution uniqueness. The left hand plot is the solution space when the solution for an event is constrained with P- and S-wave arrival time picks from all three observation wells plus hodograms from CCS1 and VW2. The right hand plot is the solution space for same event when constrained using only time picks and hodograms from CCS1. The real solution minima becomes much more clearly defined because of the improved observation geometry. 


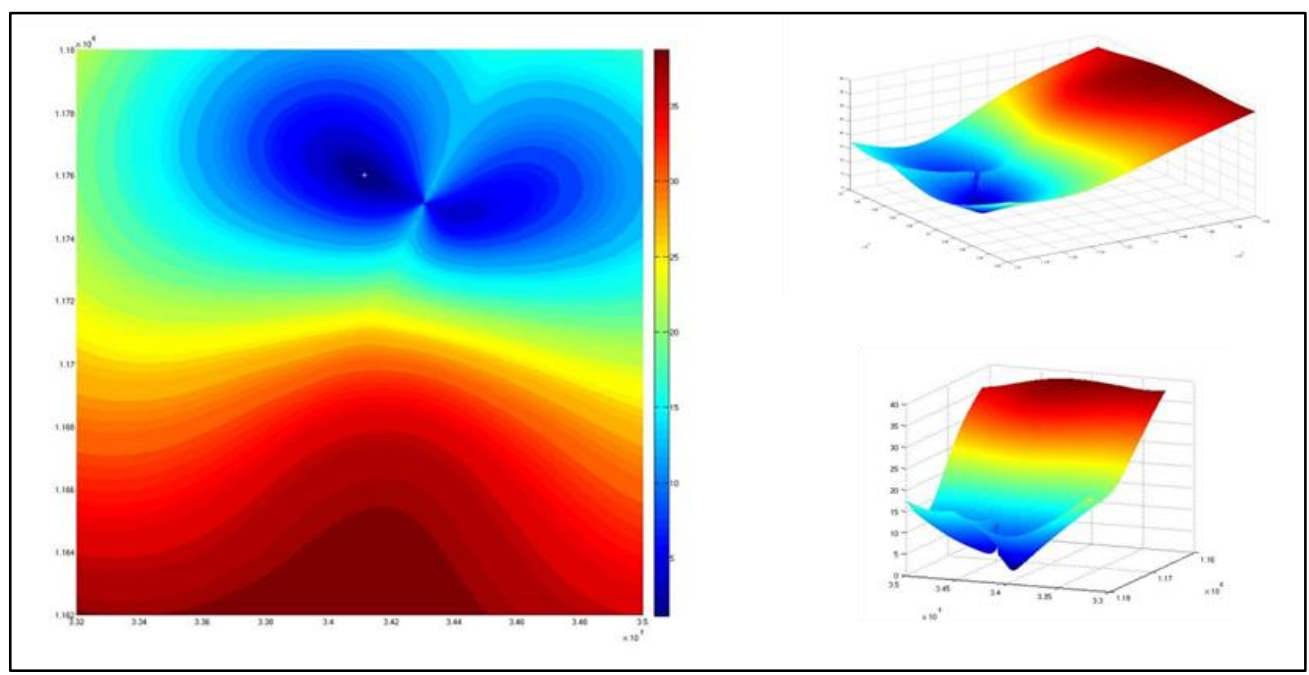

Figure 14 - Geiger solution RMS residual slice for a selected event near well VW2 exhibiting two strong minima.

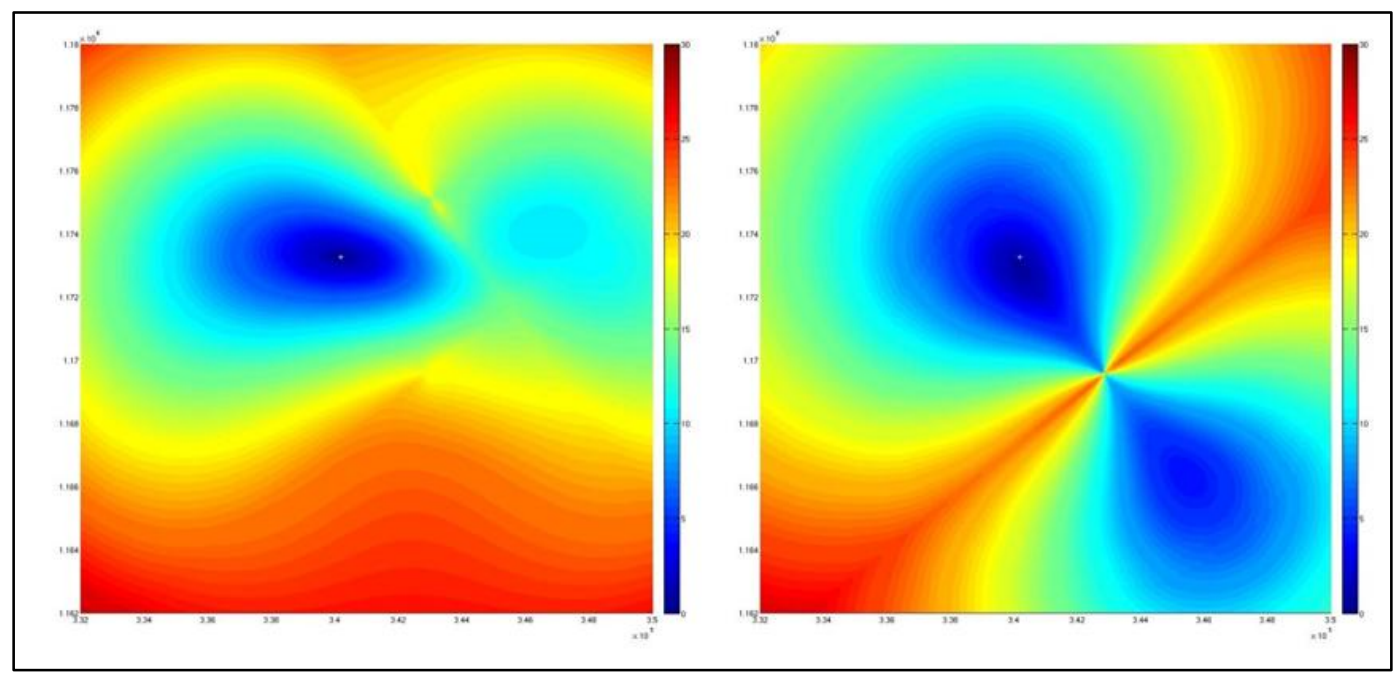

Figure 15 - (Left) Geiger solution RMS residual slice for a selected constrained with P- and Swave arrival time picks from all three observation wells plus hodograms from CCS1 and VW2. (Right) the same event using only time picks and hodograms from CCS1.

Figures 16 and 17 show the locations of all events acquired from the start of injection up to and after addition of the array in VW2 to the monitoring network respectively. In light of the solution, the non-uniqueness described above and the similarity of locations before and after the improvement of observation geometry that was provided by the introduction of VW2 data demonstrates the robustness of the solution technique. 

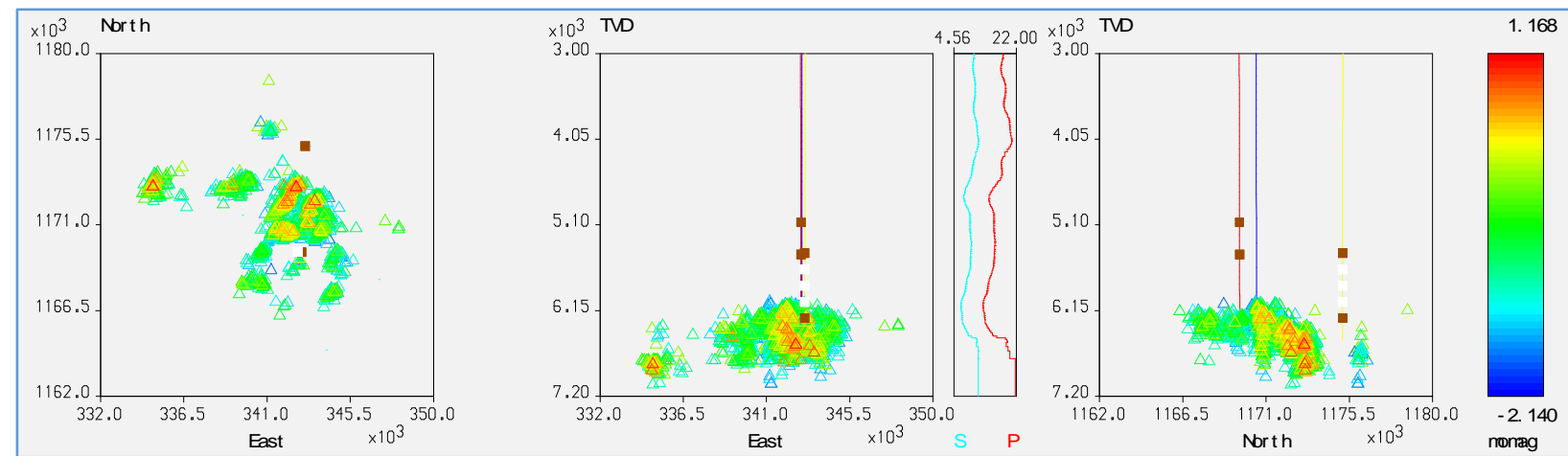

Figure 16 - Locations of 3,413 located events since start of injection
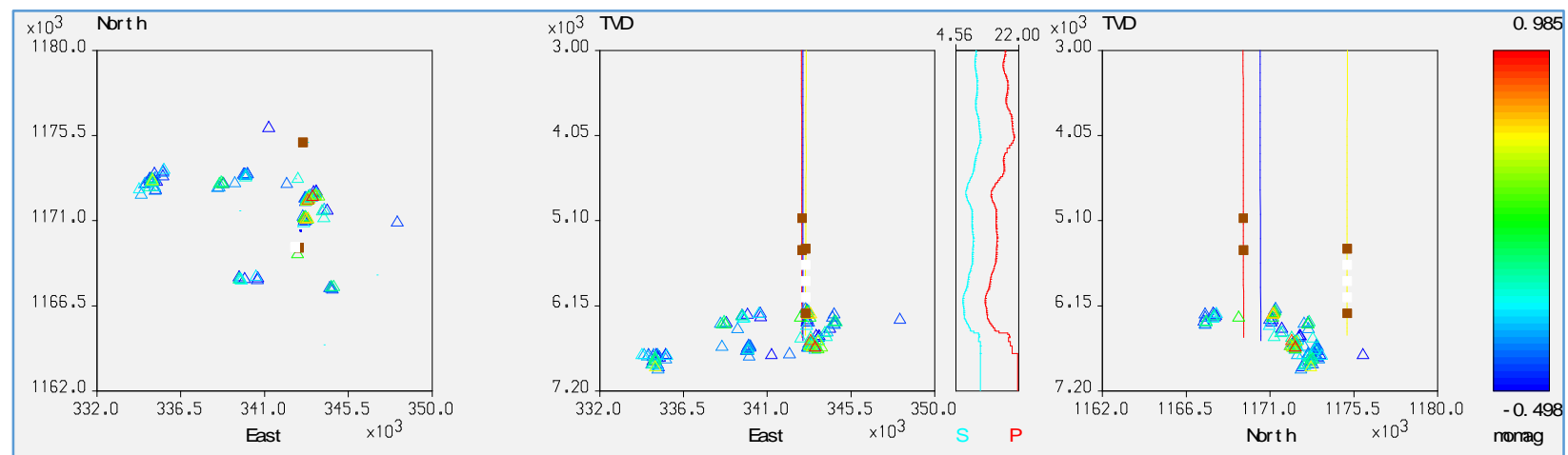

Figure 17 - Locations of 118 events with magnitude $>-0.5$ since installation of the 5-level array in VW2

\section{Source Characterization}

\subsection{Magnitude}

Magnitudes were computed for all located events using displacement spectral analysis techniques first developed by Brune et al (1970). Brune shows that, by approximating a double coupled displacement as a circular feature of equivalent surface area, analytical solutions for focal characteristics may be derived based on characteristics of the radiated seismic energy. Using this formulation event magnitude, stress drop, and displacement area may be computed from zero frequency amplitude $\left(\square_{0}\right)$ and corner frequency $\left(f_{c}\right)$ of the recorded displacement spectrum. Figure 18 shows an example of the displacement spectrum with $\square_{0}$ and $f_{c}$ picks for an event recorded at the CCS1 array.

A microseismic activity summary report is prepared on a monthly basis as an aid to rapid evaluation of recent microseismic activity as well as providing a tool for detecting changes in spatial or temporal trends. Examples of event magnitude from the IBDP monthly summary report are shown in Figures 19 through 21.

The orientation of linear clusters observed in Figure 19 is generally consistent with that expected for tectonic events within the known in-situ stress field. These features are the focus of FPS analysis discussed in the following section; however, in general, the largest observed magnitudes are located along trends which correspond to the highest critical stress condition as determined through geomechanical modeling. Other graphical and statistical analyses reported on a monthly basis include; frequency distributions of event magnitude for the current month, the previous 
month, and the prior three month average (Figure 20), plots of the relationship between event magnitude and distance from the injection well (Figure 21), and tabulation of the associated event magnitude statistics (Table 3). From these summary materials any variations in magnitude over time for the large data volume are easily discernable.

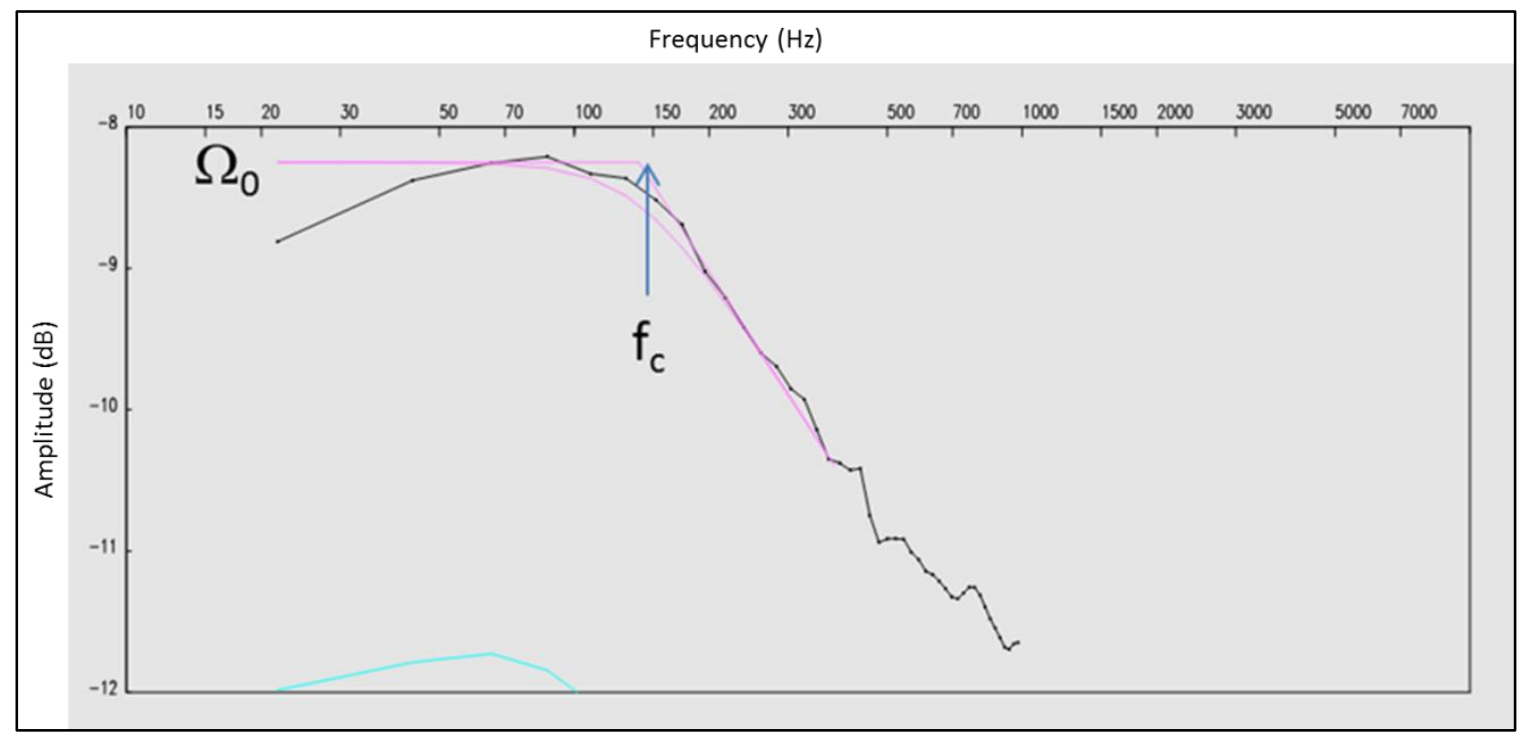

Figure 18 - Sample spectral analysis of data from CCS1 used to compute event magnitude. Geophone spectra (black), best model fit (magenta), corner frequency $\left(f_{c}\right)$ and zero frequency amplitude $\left(\Omega_{0}\right)$.

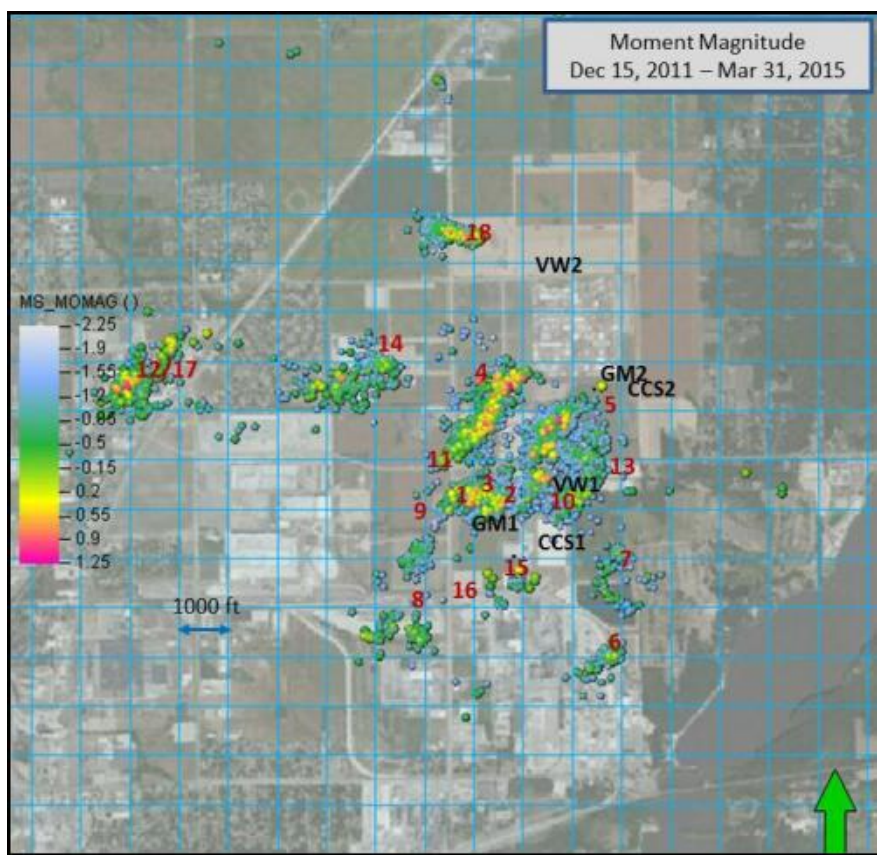

Figure 19 - All events characterized from the start of injection to end of March 2015, color scale is moment magnitude computed using displacement spectral analysis. 


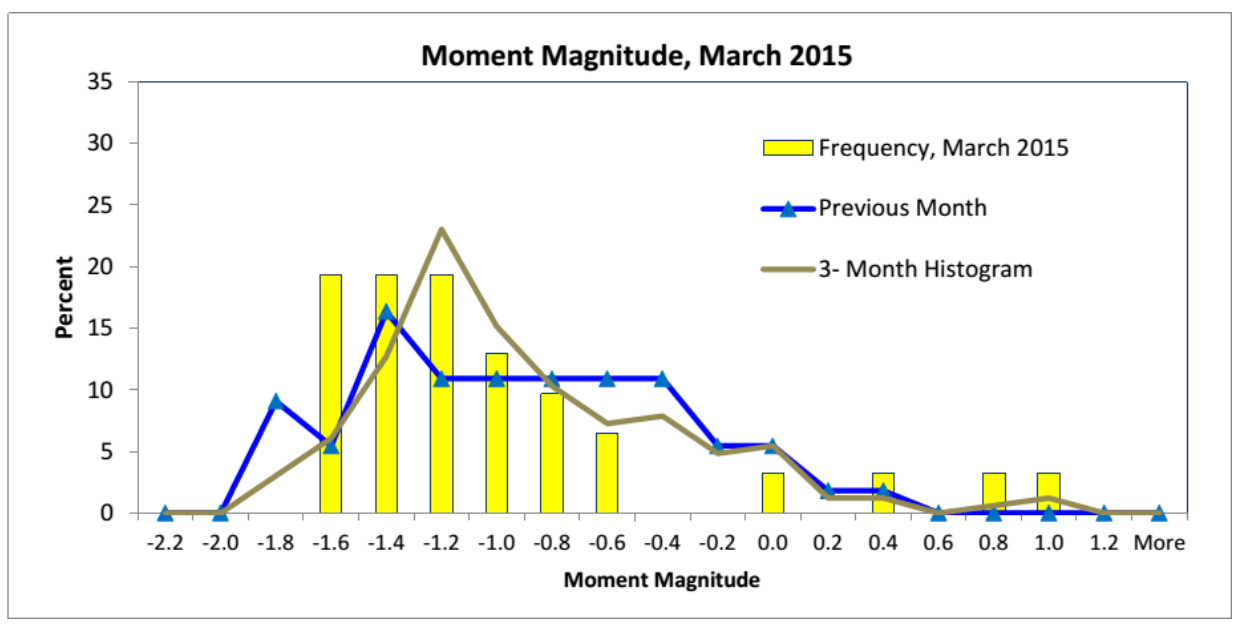

Figure 20 - Example of monthly summary showing magnitude histogram for current month and historical event magnitudes.

\begin{tabular}{|c|c|c|c|c|c|}
\hline & Detected & Located & Mean & StDev & Max \\
\hline March 2015 & 67 & 31 & -1.09 & 0.68 & 0.87 \\
\hline Previous Month & 136 & 50 & -0.95 & 0.53 & 0.25 \\
\hline 3-Month Average & 189.0 & 78.0 & -1.02 & 0.57 & 0.64 \\
\hline
\end{tabular}

Table 3 - Example of event statistical summary for current reporting month.

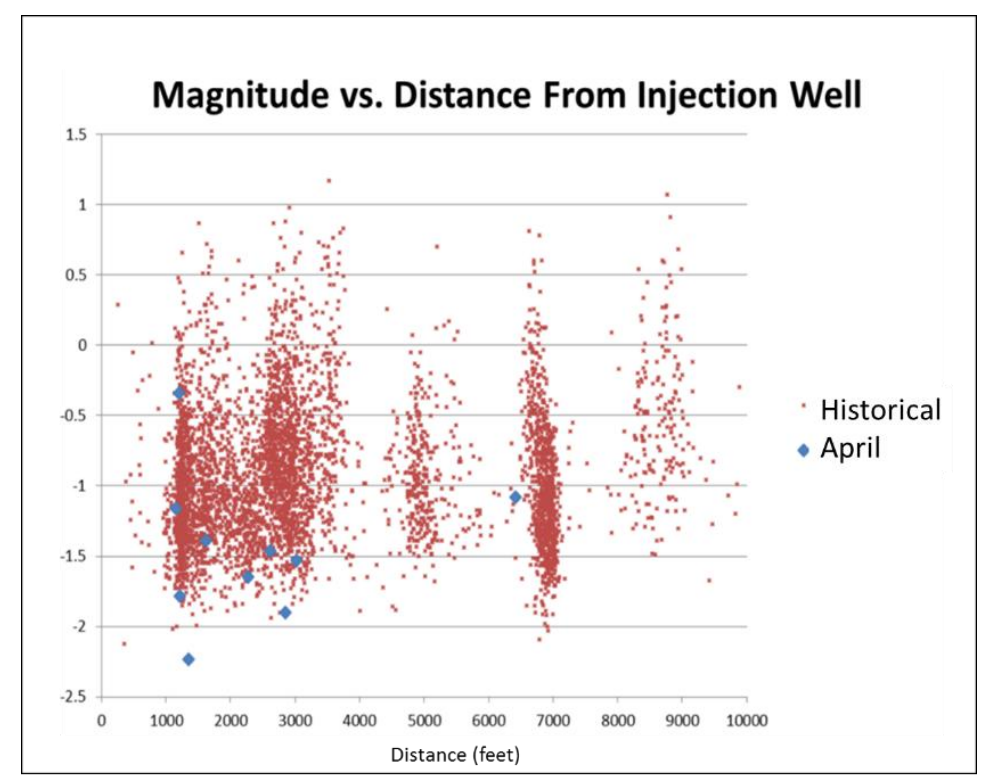

Figure 21 - Excerpt from monthly summary report showing event magnitude versus distance from the injection well. The blue diamonds designate activity occurring during the given reporting month. 


\subsection{Fault Plane Solutions}

The FPS analysis was performed on the located events shown in Figure 23 using a strike-slip focal mechanism model suggested by the in-situ stress tensor obtained through geomechanical characterization. The methodology for characterization of fault planes using $\mathrm{P}$ and $\mathrm{S}_{\mathrm{h}}$ amplitude ratios is described by Shi et al (2009) and involves fitting of an analytical solution of P and horizontal Shear $\left(\mathrm{S}_{\mathrm{h}}\right)$ amplitudes for a group of events believed to share a common focal mechanism. The events shown in Figure 22 clearly exhibit linear clusters suggesting several individual focal mechanisms. An FPS analysis was first performed on all events together. Figure 22 shows the result of the FPS analysis for average azimuth computed from all events using a fault plane azimuth of $\mathrm{N} 20 \mathrm{E}$. The data shows a reasonable fit to this azimuth with some dispersion, indicating that not all events belong to the same source mechanism. Figure 24 shows the FPS solution for inclination of all events taken together using an average azimuth of N20E. These events show a reasonable fit to the model assumptions with dispersion in inclination possibly resulting from either real dispersion in the inclination of features, or non-conformance to the N20E azimuth assumption used in the model.

FPS analysis was then performed on data after sorting into individual spatial clusters. Figure 25 shows the FPS result for the most developed and coherent cluster exhibiting a very good fit to the strike-slip model for an azimuth of N45E. The results of FPS analysis for all clusters are shown in Figure 27. These orientations were used to define source mechanisms in later numerical hydro-mechanical modeling. The use of FPS analysis was supported by the RichterGutenburg analysis (Gutenberg et al, 1956) shown in Figure 28 which shows a slope of 1, indicating a tectonic event mechanism rather than injection induced fracturing (Stein and Wysession, 2003, Downie et al, 2010).

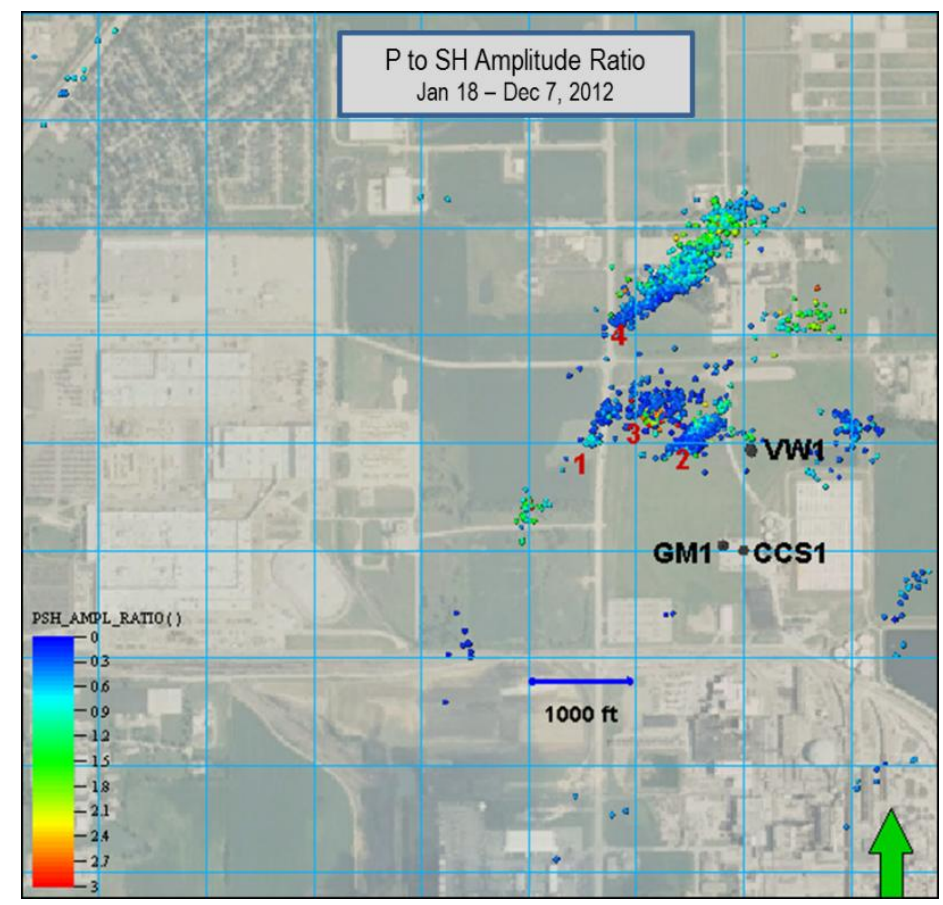

Figure 22 - Events used for FPS analysis. Color scale is $\mathrm{P}$ to $\mathrm{S}_{\mathrm{H}}$ amplitude ratio. 


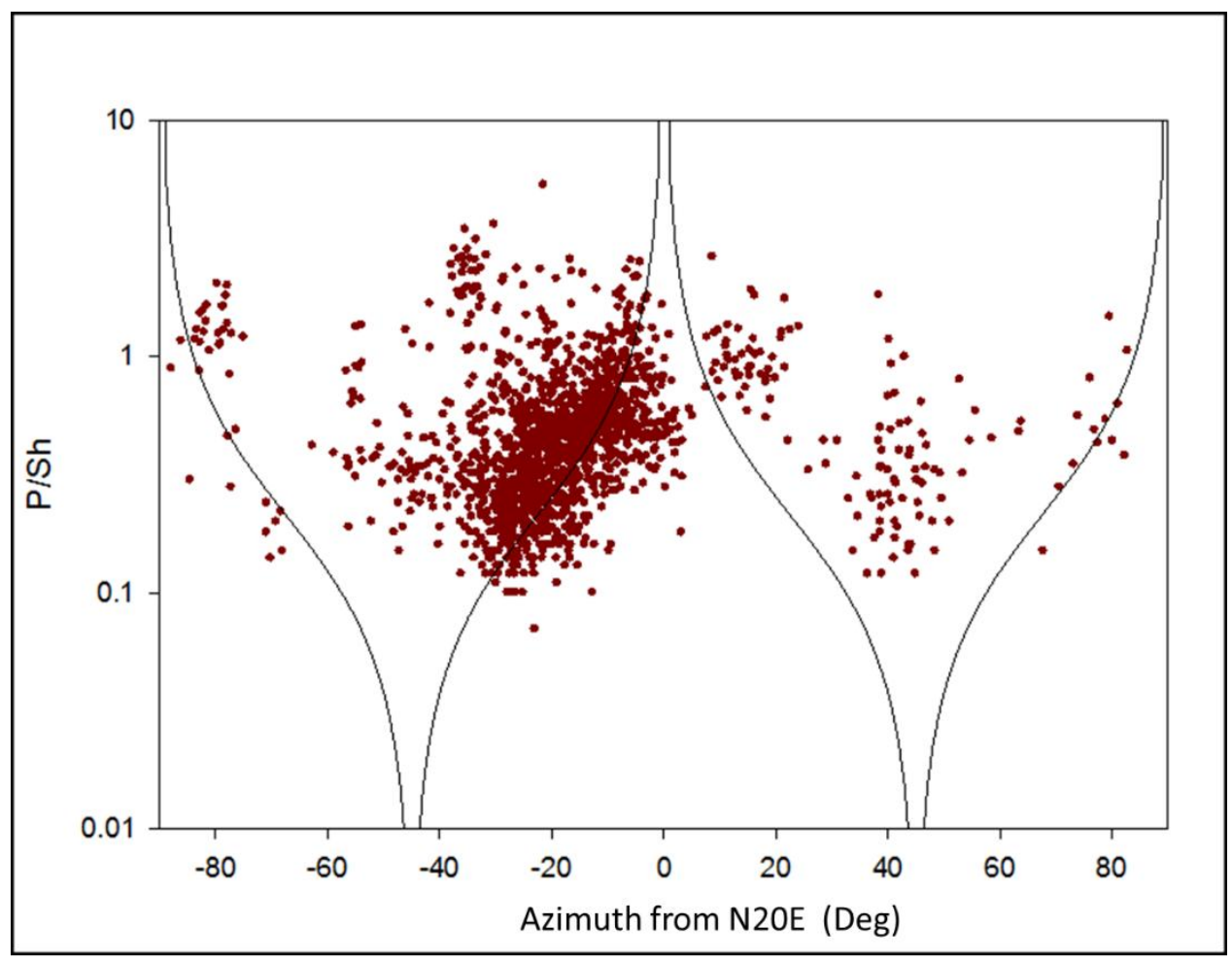

Figure 23 - Fault plane solution for all events using a strike-slip assumption and average azimuth of N20E.

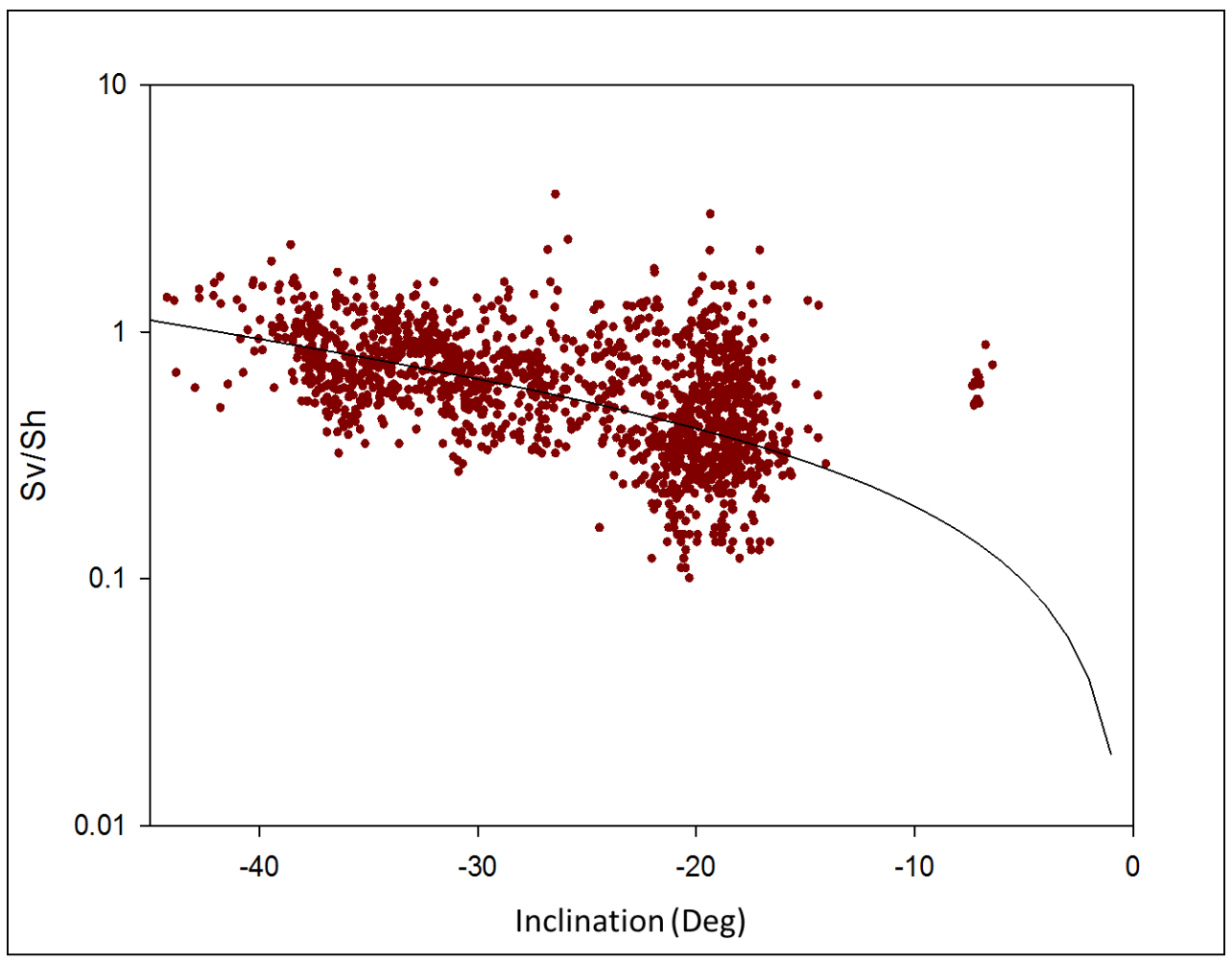

Figure 24 - FPS inclination analysis using strike slip model with average azimuth of N20E. 


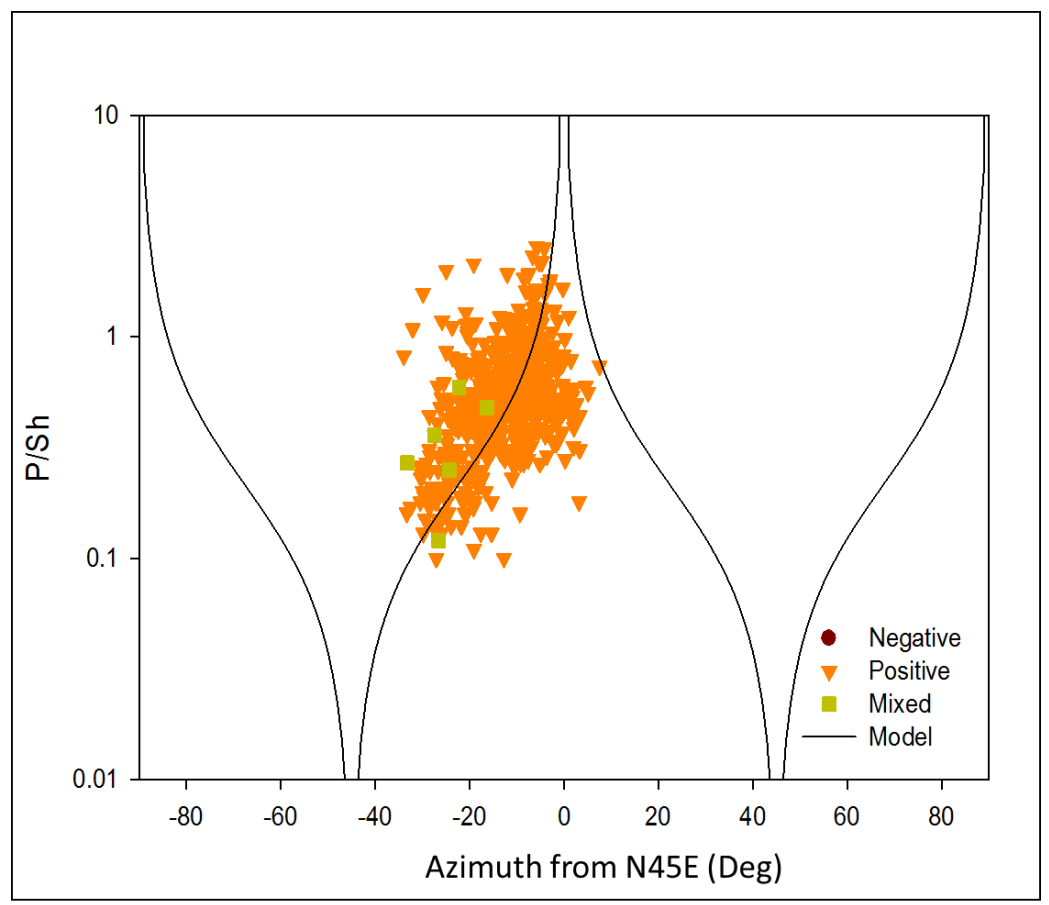

Figure 25 - FPS for selected event cluster (4) using a strike-slip assumption and azimuth of N45E.

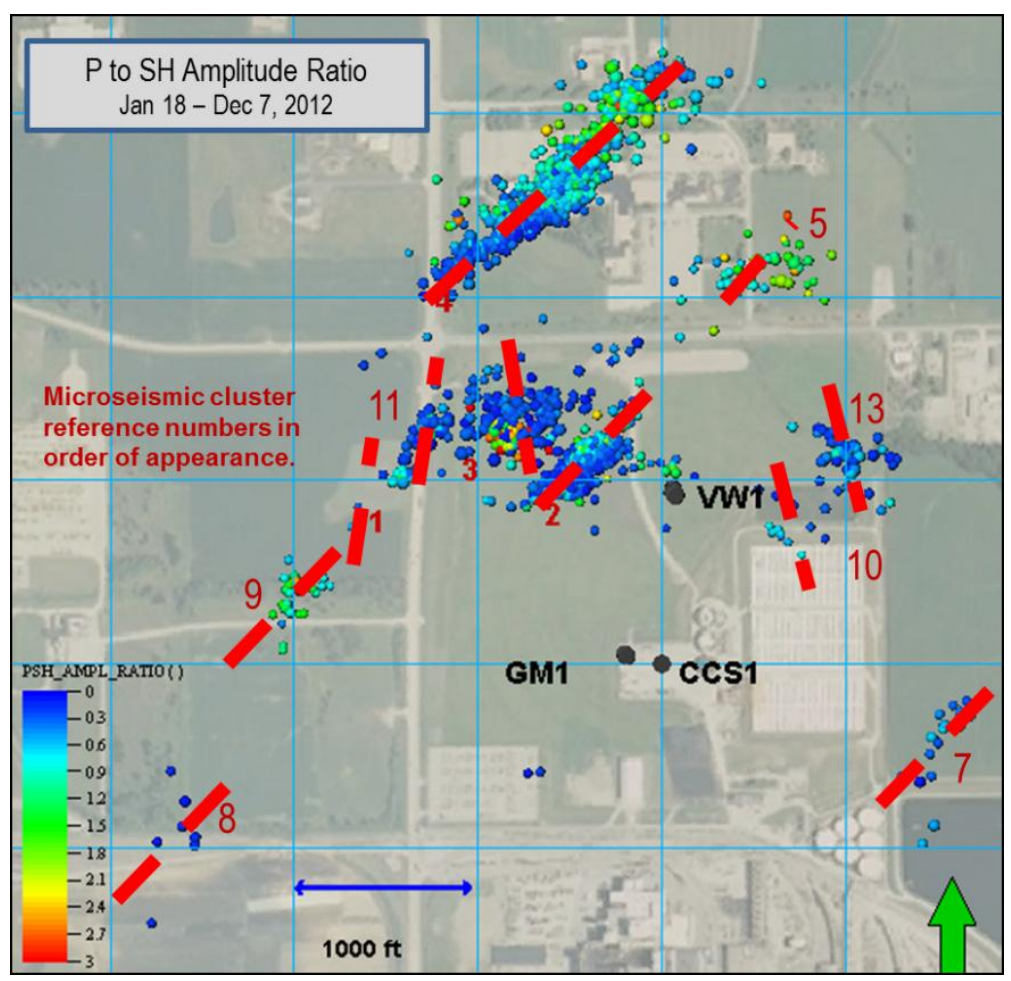

Figure 26 - FPS results for all clusters. 


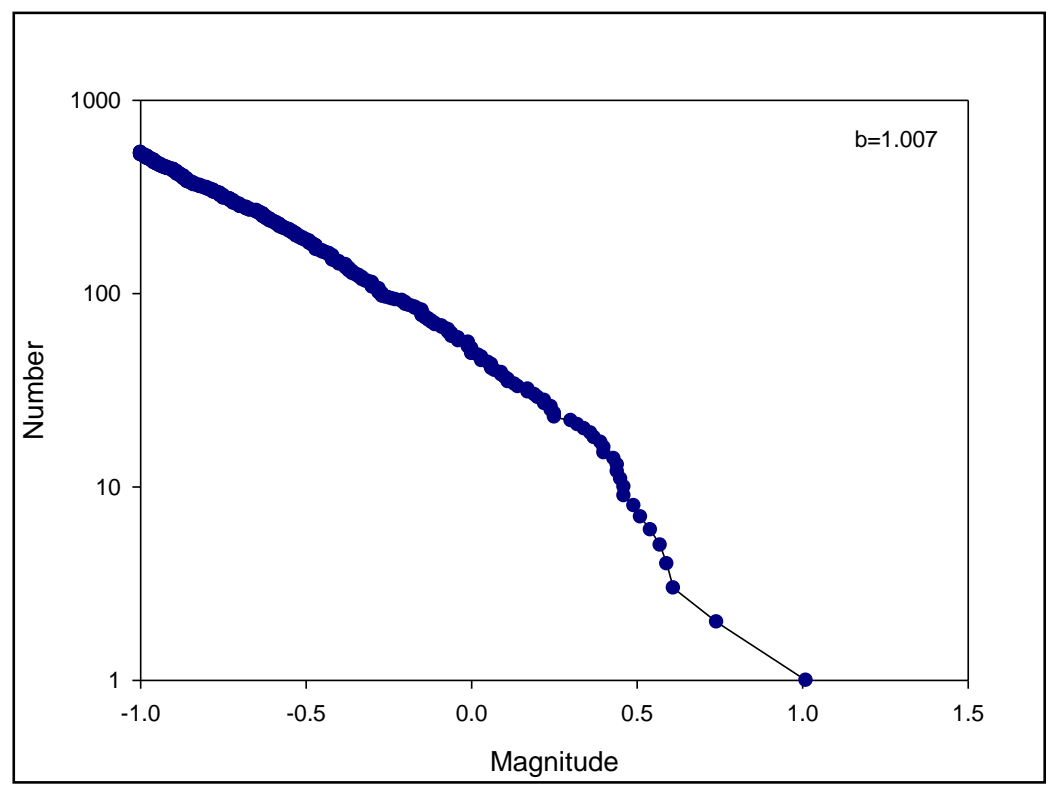

Figure 27 - Richter-Gutenberg analysis of events used in FPS solutions

\subsection{Other Trend Analysis}

In addition to the statistical analysis of magnitude data shown in Figures 20 and 21, the monthly report includes various other analyses designed to assist in the detection of changes in spatial trends over time. Figure 28 shows an example of daily rates and cumulative numbers of detected and located events. Figure 29 illustrates the spatial distribution of located events from a perspective that highlights vertical distribution. New events for the month are annotated in yellow. Figure 30 shows an analysis of the temporal distribution of all events to date throughout the local week and time of day (morning/night).

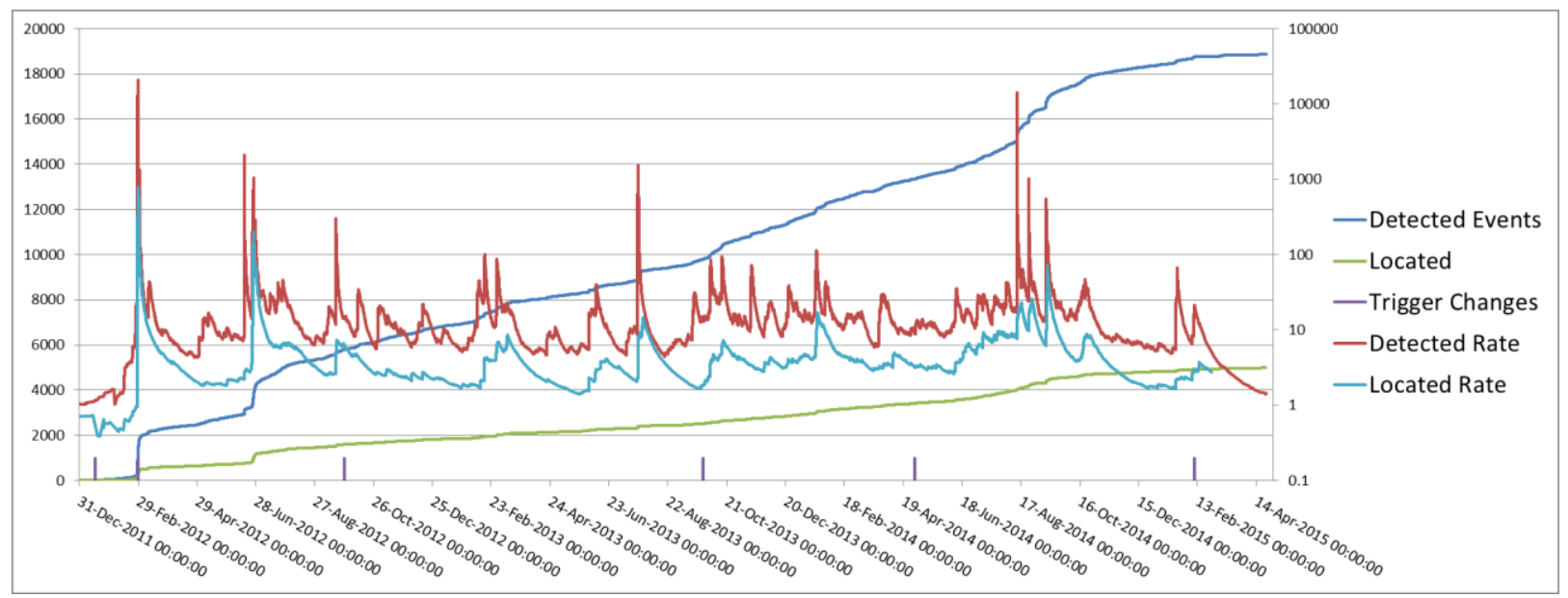

Figure 28 - Excerpt from monthly summary report showing daily and cumulative event statistics. 


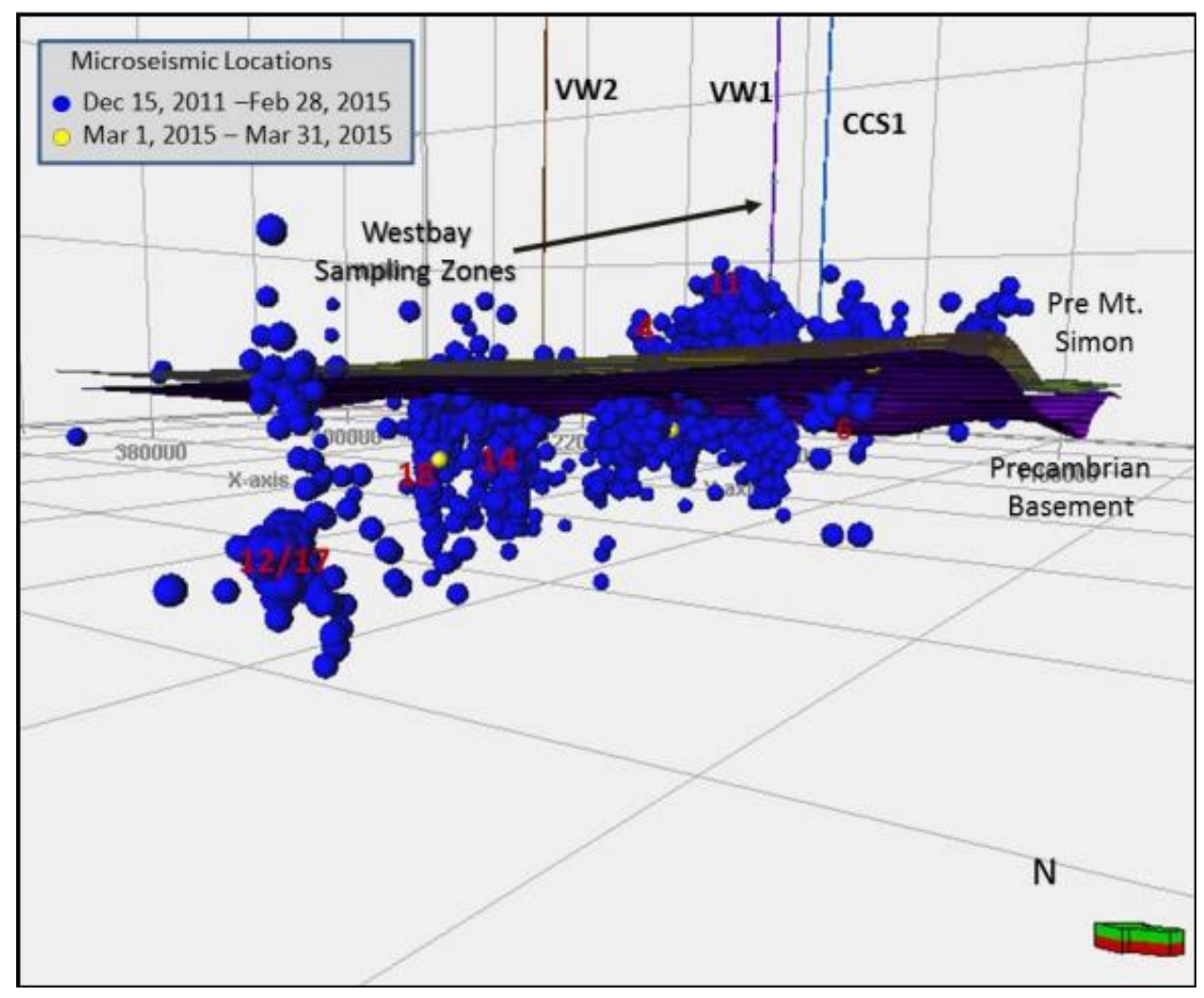

Figure 29 - Excerpt from monthly summary report showing vertical distributions of all events located to date.

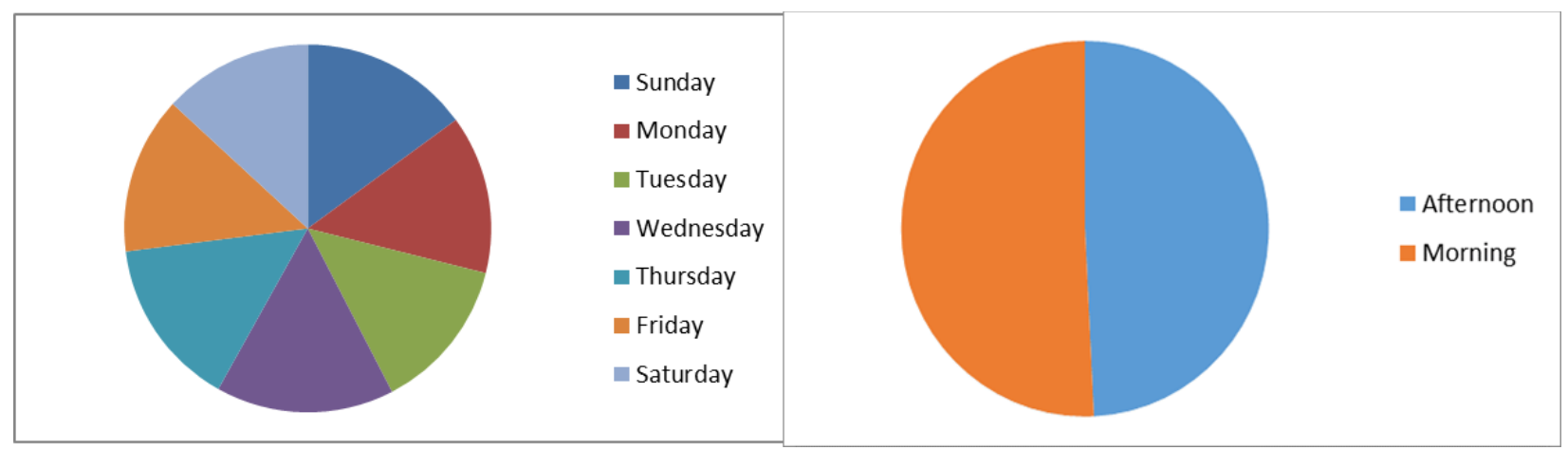

Figure 30 - Excerpt from monthly summary report showing daily occurrence rates of all located event activity.

\section{Tool Orientation and Velocity Model Calibration}

\subsection{Tool Orientation}

The fundamental need to properly orient microseismic events in 3D space requires precise knowledge of the position of the geophones in $\mathrm{X}, \mathrm{Y}$, and $\mathrm{Z}$ directions and the orientation of the geophones within that position. The geophone orientation procedure involves initiation of an 
energy source, such as a perforation gun shot or seismic vibrator, at a known location. Analysis of recorded particle motion (hodograms) from these shots defines a vector which should "point at" the known source location. The receiver orientation is done by computing a relative bearing angle (RBG) correction for each receiver. The RBG correction takes into account the borehole azimuth and deviation, the measured polarization estimated from the hodogram analysis around the P-wave arrival time for each receiver, and the source-receiver vector.

Tool orientation calculations were performed on the various IBDP subsurface arrays at different times during the monitoring project as new subsurface components were added and energy sources became available as a result of field activities. Since the array in CCS1 provides the primary observations constraining the azimuth used in calculating micro-seismic event locations, verifying accurate orientation of these geophones was attempted during all orientation efforts.

\subsection{Chronology}

January 2010: Initial receiver orientation was performed using the 3D VSP source locations. Although the signal-to-noise ratio was low, reasonable orientation was achieved (within +/- 3 degrees of further orientation methods).

January 2102: $\quad$ Orientation was performed using eleven perforation shots fired in VW1 in May 2011. The sampling rate of two milliseconds used in recording waveform data for estimation of gun firing time was not ideal; however, the test was deemed sufficient to obtain an improved orientation for the CCS1 receivers.

November 2012: Perforation shots were performed in VW2 during the well drilling for the purpose of helping to fine tune GM1 and CCS1 geophone orientations and verify the velocity model. Due to low signal-to-noise ratio, the hodograms were deemed unsuitable for use in computing tool orientations. A distant earthquake identified in the USGS database provided some degree of confirmation that the geophone orientations were accurate.

January 2013: During the interim period after installation of the retrievable array in VW2, but before an energy source could be deployed for tool orientation, preliminary orientations for the VW2 array were estimated using microseismic events with locations estimated using CCS1 and GM1 arrays.

February 2014: Selected seismic vibrator source points from the 3D VSP acquired at GM1 were used to compute tool orientations for all arrays. Twenty vibroseis sweeps acquired at each of fourteen surveyed locations were summed to provide good signal fidelity for orientation calculations. Figure 31 shows the locations of vibrator sweeps used for the February 2014 receiver orientation calculations. The example summed sweep from the VW2 array shown in Figure 32 exhibits clear P- and S-wave arrivals and linear particle motion hodograms for all tools.

The three arrays are oriented whereby azimuth is the angle between the North and tangent to the well projected on horizontal plane, measured clockwise from North. Deviation is the angle of the 
local well trajectory to the vertical. Relative bearing is the angle in the inclined plane around the inclined axis parallel to the borehole deviation.

Due to the shallow deployment depth of the GM1 array, orientations of these tools could not be estimated with an adequate degree of azimuthal uncertainty. As a result, only P- and S-wave arrival times from this array are used for event locations.

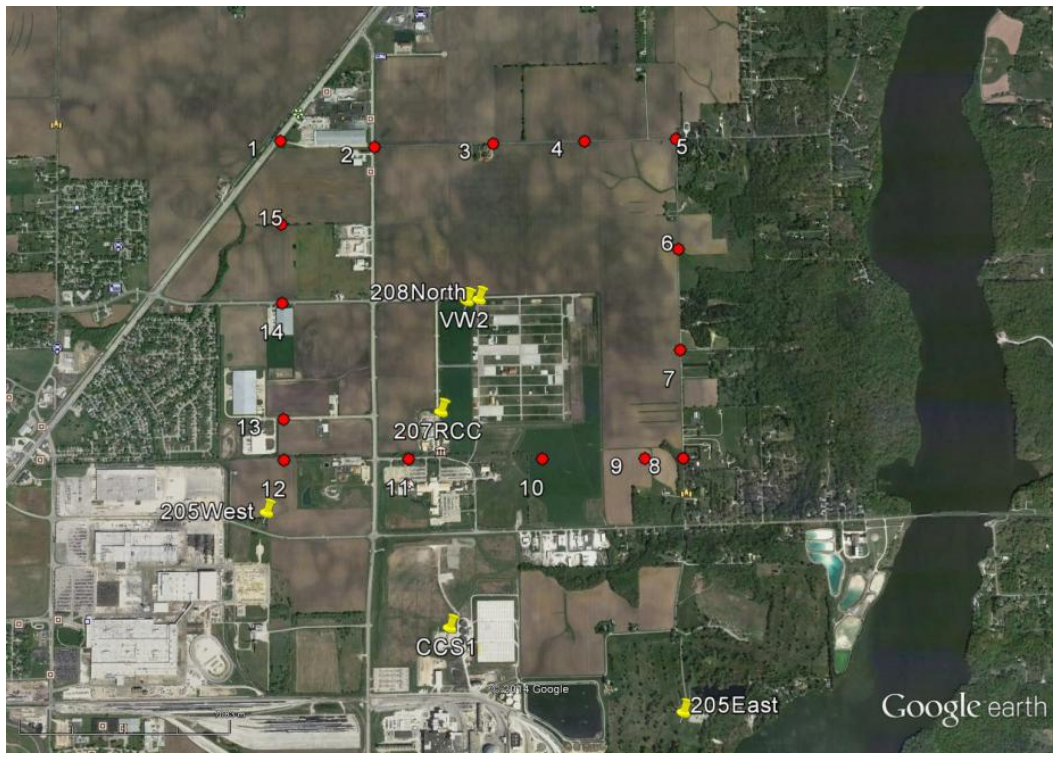

Figure 31 - Vibrator station locations for February 2014 receiver orientation data acquisition.

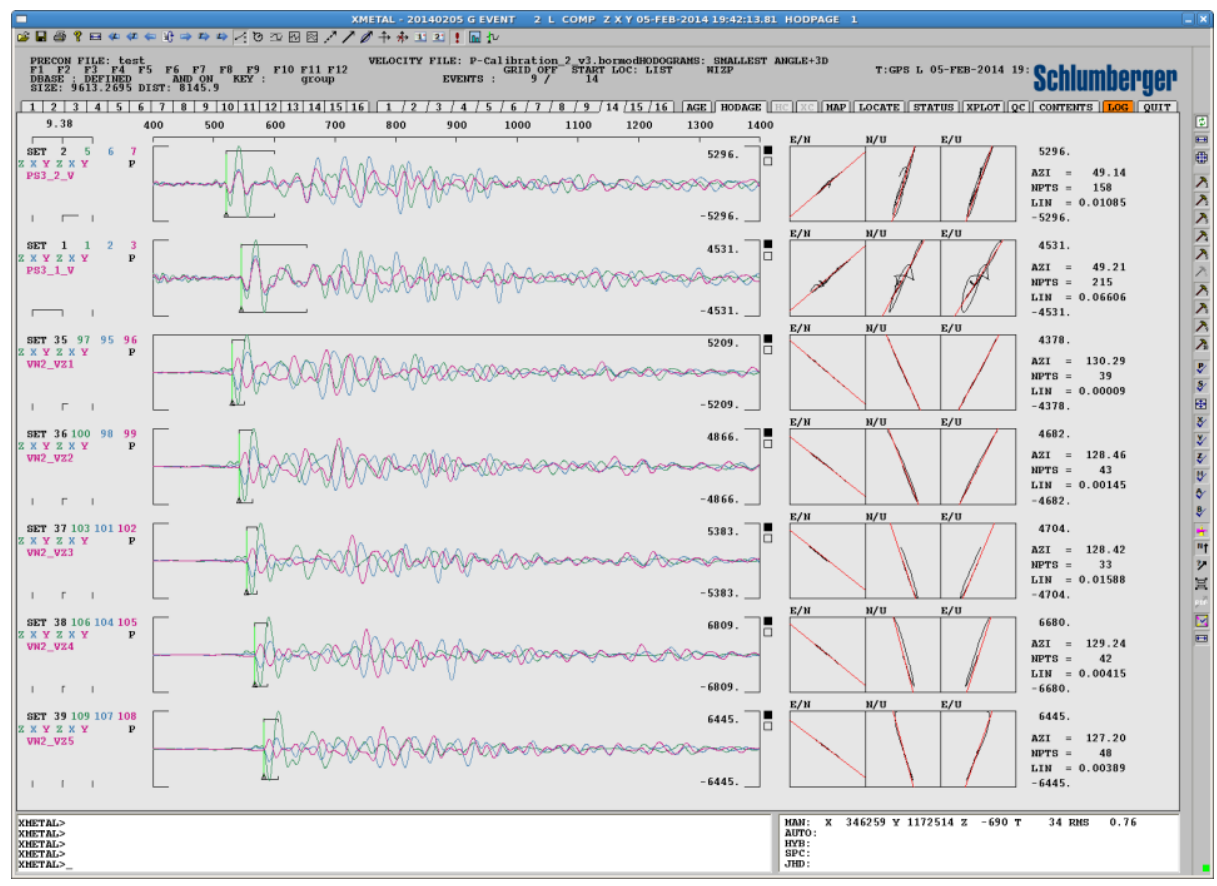

Figure 32 - Seismograms and hodograms from the five VW2 tools for a sample VSP orientation shot. 


\subsection{Velocity Model Calibration}

\section{Background}

Multiple versions of the velocity model were used for microseismic event location and characterization at IBDP throughout the life of the project. The Velocity Model updates were driven by two main factors;

1. The evolution of microseismic activity away from the injector well as the plume and pressure front expanded which resulted in anomalous location solutions.

2. The introduction of new monitoring components provided the opportunity for a more robust calibration.

Successive versions of the velocity model were created using different subsurface network components and different controlled energy source types. In addition to the difference is the field data used for calibration, these models have contained various representations of anisotropy and differing vertical extent.

\section{Model Structure}

The velocity model used for event location and characterization at IBDP is a one-dimensional layer cake structure such that interval velocity varies with depth but is laterally constant within each layer. All model versions have been initialized using compressional and shear sonic logs acquired in injection and monitoring wells. Anisotropic model versions utilize Thomsen's VTI formulation (Thomsen, 1986). VTI parameters are constant within each model layer.

\section{Model Revisions}

The initial velocity model was defined by sonic logs from CCS1 with VTI calibrated using surface seismic vibrator sweeps and perforation gun shots in VW1. This VTI characterization was later deemed to be unstable and subsequently removed from the model. As injection continued and fluid volume increased, the observed microseismicity progressed to greater distances from the injection well. An observed systematic shift in depth of these distal events prompted review of the velocity model and resulted in the addition of deeper layers to represent the Pre-Cambrian formation, followed by the recalculation of VTI. VTI calibration was performed by two independent analysts, yielding two independent VTI formulations. When applied to the existing dataset both VTI models result in event locations farther from the injector well (Figure 33), and deeper (Figure 34) compared to those computed using the isotropic model. Anisotropic velocity model 1 (red) locates events furthest from CCS1 and deepest while anisotropic velocity model 2 (yellow) locates events at intermediate distance and depth between the isotropic model (magenta) and anisotropic model 1.

\section{Results Comparison and Final Model Selection}

While deployment of the 5-level array and the acquisition of perforation gun energy source points in VW2 provided much needed constraints on the calculation of VTI parameters, these 
supplemental data also yielded the opportunity to compute a number of additional metrics for assessment of the "best" VTI parameters.

The following three metrics were used to compare the two independent VTI formulations;

1. The WellWatcher PS3 P-wave residual RMS values from VW2 perforation shots

2. The WellWatcher PS3 P- and S-wave residual RMS values for a selection of (23) events with magnitude greater than 0.5 and location between wells CCS1 and VW2.

3. P- and S-wave residual RMS values from the WellWatcher PS3 and VW2 tools for a selected co-located (VW2 using observations from both arrays) event located between CCS1 and VW2.

Anisotropic model 2 was selected based on best performance against all three metrics. As a result of this assessment all events to date (including those prior to the assessment) have been located using anisotropic velocity model 2.

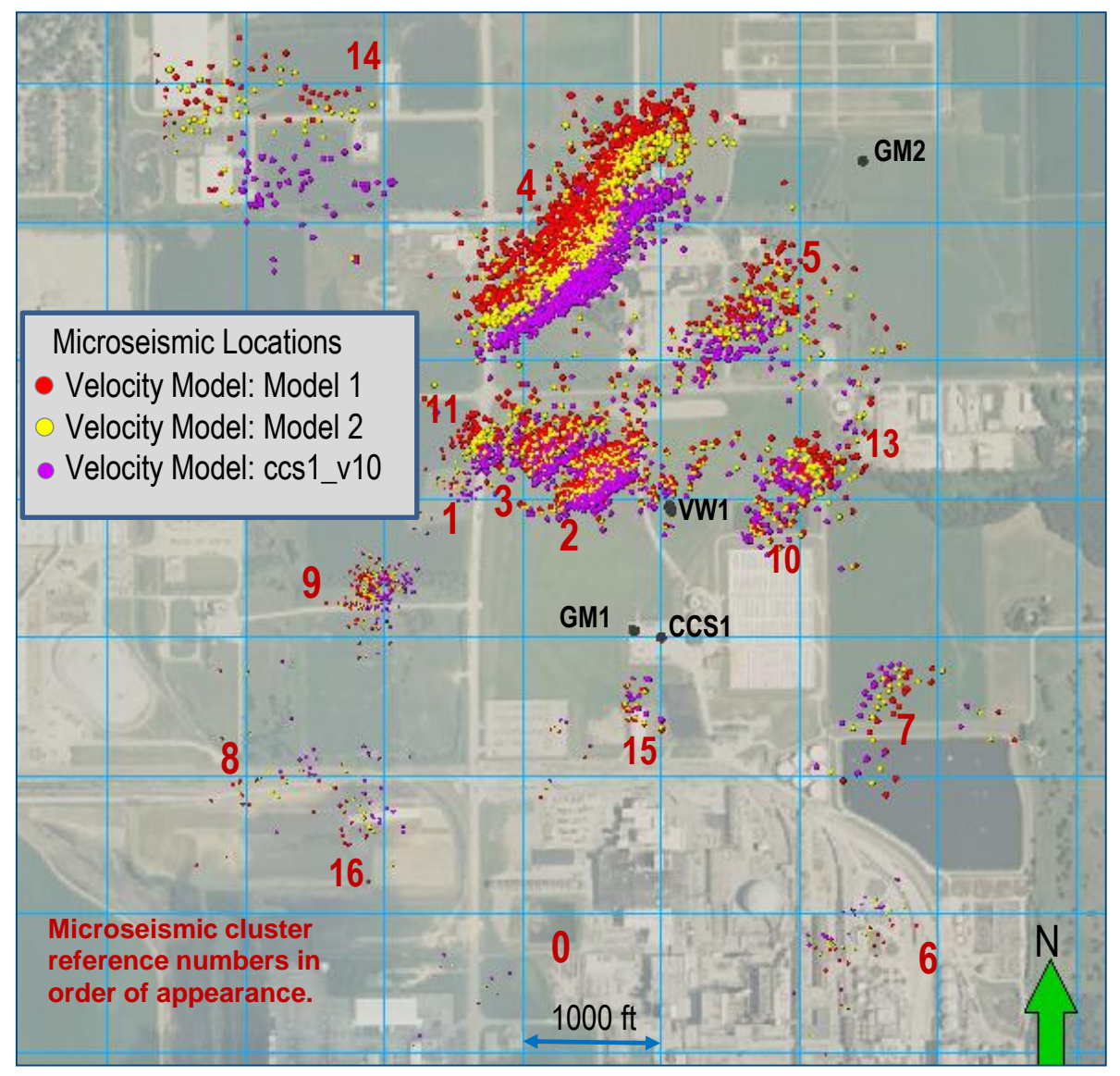

Figure 33 - Map view of microseismic event locations computed using two different anisotropic models (Model 1 and Model 2) and isotropic model (ccs1_v10). 


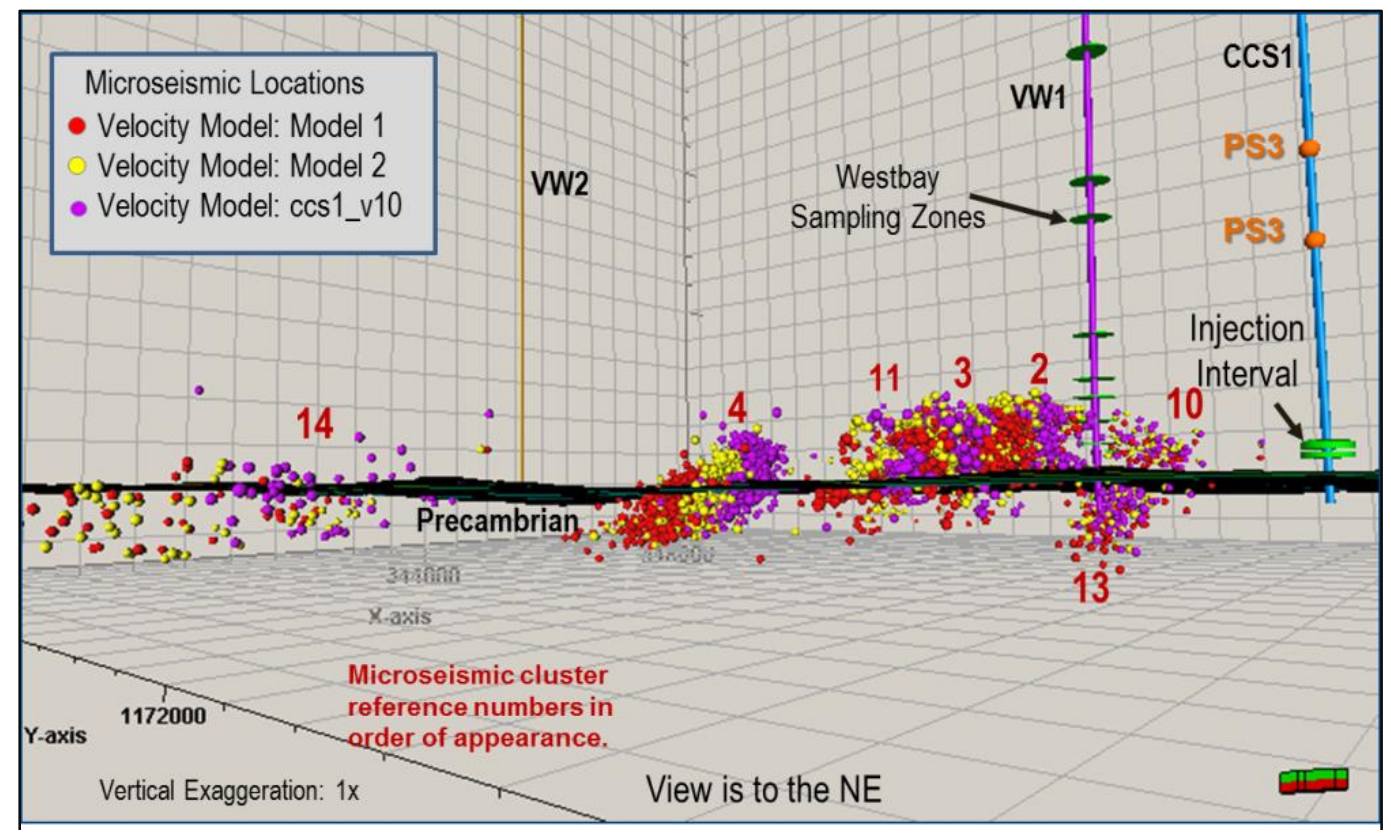

Figure 34 - Side view of microseismic event locations computed using VTI Model 1 (red), VTI Model 2 (yellow), and isotropic model (magenta).

\section{Summary}

Each day approximately $70 \mathrm{~GB}$ of data are recorded from a combination of three permanent and retrievable subsurface arrays at IBDP and integrated at the field site by means of an on-site data management and computing system. Automated real-time and limited manual in-field event triggering reduces the volume of raw data required for in-house analysis to levels efficiently transferred via remote connection. Initial in-house processing steps further reduce data volume for final analysis. These combined in-field and in-house data preparation steps result in nearly a $99 \%$ reduction in the amount of data input to the event location and focal mechanism characterization workflow.

Event location is performed using a modified form of Geiger's method which is designed to treat the common problem of location solution non-uniqueness in sparse observation networks. Comparisons facilitated by the deployment of a supplemental subsurface array, strengthening observation network geometry and redundancy, demonstrate the robustness of this location method for the IBDP network. Linear spatial trends observed in microseismic locations suggest tectonic focal mechanisms consistent with the local in-situ stress regime. Focal mechanism characterization performed using standard techniques including Richter-Gutenberg, displacement spectral analysis, and fault plane solution analysis support the interpretation of numerous discrete event sources which have been used in mechanical earth modeling efforts.

Event locations and focal mechanism computations utilized a layer cake velocity model, multiple versions of which have been developed in response to the addition of new network components and evolving spatial distribution of microseismicity. An evaluation of the velocity model, prompted by the observation of an evolving trend in event depth versus distance from the 
injection well, informed modifications to the velocity model in terms of basement structure velocities and anisotropy characterization.

The IBDP microseismic monitoring effort represents successful application of commercially available and purpose-built technology under challenging operational conditions to achieve difficult technical objectives. Equally as important as this technical success is the demonstration of a system with the flexibility and robustness that will be required for large-scale commercial applications under the anticipated regulatory environment.

\section{Acknowledgement}

This work was conducted under the Midwest Geological Sequestration Consortium which is funded by the U.S. Department of Energy through the National Energy Technology Laboratory (NETL) via the Regional Carbon Sequestration Partnership Program (contract number DE-FC2605NT42588) and by a cost share agreement with the Illinois Department of Commerce and Economic Opportunity, Office of Coal Development through the Illinois Clean Coal Institute.

\section{References}

Brune, J. N. (1970). Tectonic stress and the spectra of seismic shear waves from earthquakes, J. Geophys. Res. 75, 4997-5009

Downie, R.C., Kronenberger, Maxwell, S., Using Microseismic Source Parameters to Evaluate the Influence of Faults on Fracture Treatments - A Geophysical Approach to Interpretation, SPE 134772, Presented at the SPE Annual Technical Conference and Exhibition, Florence, Italy, 1922 September, 2010.

Geiger, L. (1912). Probability method for the determination of earthquake epicenters from the arrival time only, Bull. St. Louis Univ. 8, 60-71.

Gutenberg, B., Richter, C. F., 1956. Magnitude and Energy of Earthquakes. Annali di Geofisica, 9: -15

Shi, Z. and Y. Ben Zion (2009), Seismic radiation from tensile and shear point dislocations between similar and dissimilar solids, Geophysical Journal International 179 (1), 444-458. Stein and Wysession; An Introduction to Seismology, Earthquakes, and Earth Structure, 2003, Blackwell Publishing.

Thomsen, Leon (1986). "Weak Elastic Anisotropy". Geophysics 51 (10): 1954-1966. 